Research Article

\title{
Numerical Investigation and Design of Reinforced Concrete Shear Wall Equipped with Tuned Liquid Multiple Columns Dampers
}

\author{
Zhe Wang $\mathbb{D}^{1},{ }^{1}$ Liang Cao $\mathbb{D}^{\circ},{ }^{2}$ Filippo Ubertini $\mathbb{D}^{3},{ }^{3}$ and Simon Laflamme $\mathbb{D}^{1,4}$ \\ ${ }^{1}$ Department of Civil, Construction, and Environmental Engineering, Iowa State University, Ames, IA, USA \\ ${ }^{2}$ ATLSS Engineering Research Center, Lehigh University, Bethlehem 18015, PA, USA \\ ${ }^{3}$ Department of Civil and Environmental Engineering, University of Perugia, Perugia, Italy \\ ${ }^{4}$ Department of Electrical and Computer Engineering, Iowa State University, Ames, IA, USA \\ Correspondence should be addressed to Zhe Wang; zhew@iastate.edu
}

Received 13 November 2020; Revised 12 January 2021; Accepted 16 February 2021; Published 28 February 2021

Academic Editor: Salvatore Caddemi

Copyright (c) 2021 Zhe Wang et al. This is an open access article distributed under the Creative Commons Attribution License, which permits unrestricted use, distribution, and reproduction in any medium, provided the original work is properly cited.

\begin{abstract}
The tuned liquid multiple column damper (TLMCD) is a variation of the tuned liquid column damper (TLCD) that includes multiple vertical columns. A new damping system that embeds TLMCDs within reinforced concrete shear wall systems, termed tuned liquid wall damper (TLWD), is proposed, augmenting the traditional structural component with energy dissipation capabilities. The objective of this study is to assess energy mitigation and strength trade-offs in designing TLWDs and demonstrating the promise of TLWD systems in tall buildings through vertically distributed applications. This is done by investigating the performance of the proposed TLWD through the finite element model (FEM) of a simplified representation of a 42-story building equipped with the multifunctional component. A strength model for the TLWD is developed to empower faster performance evaluation on more complex models. Results from the FEM are used to validate the strength model and show that the model could be used conservatively in assessing strength performance. Design considerations are discussed based on the simplified representation. In particular, to improve mitigation performance while maintaining strength, it is found that a single-layer arrangement of the vertical columns is preferred, while distributing the inertia among a higher number of smaller columns. The proposed TLWD is numerically evaluated on a more realistic system consisting of a multi-degrees-of-freedom representation of the 42-story building under stochastic wind excitation. Simulation results demonstrate that the TLWD, used in a vertically distributed configuration through the building, could be used to mitigate vibrations, outperforming a traditional TLCD system with geometric constraints under 20 design wind realization. Results from the numerical simulations also confirmed the design considerations established through the simplified representation.
\end{abstract}

\section{Introduction}

High-strength and lightweight materials combined with advanced construction techniques have empowered the design of taller and more flexible high-rise structures, yet making them vulnerable to excessive motion that may cause discomfort during daily operations and safety concerns against natural hazards [1]. A solution is the integration of supplemental damping strategies, including passive, semiactive, and active systems [2]. Amongst these strategies, passive systems are now widely accepted by the civil engineering community due to their low cost and known long-term mechanical robustness and reliability.

Of interest to this paper is the use of tuned mass dampers (TMDs), a vibration mitigation system that dissipates energy through inertia [3]. The tuned liquid column damper (TLCD) is a variation of TMD proposed by Sakai et al. $[4,5]$ that consists of a partially water-filled U-shaped tank and an internal orifice to generate hydrodynamic head loss in addition to the internal surface friction while liquid sloshing occurs [6]. The technology is considered as an attractive alternative due to its ease of installation, low maintenance requirements, 
cost-effectiveness, and high mechanical robustness [7, 8]. In the last decades, the concept of TLCD was well investigated in terms of design optimization $[9,10]$, also with applications to multihazard mitigation $[11,12]$. However, TLCDs require significant space for implementation [12] and are only effective over a limited frequency range. Cao et al. studied a variation of the TLCD termed tuned liquid multiple columns damper (TLMCD), which consists of multiple vertical columns joined with a horizontal tube [13]. The TLMCD is capable of tuning at multiple frequencies and providing enhanced performance in a constrained geometry.

Recently, the authors proposed to implement a TLMCD system within a reinforced concrete (RC) shear wall, termed tuned liquid wall damper (TLWD), with the intent to improve vibration mitigation capabilities of shear wall components [13]. The advantage of the TLWD is in providing a geometrically restricted and distributed location for the integration of TLMCD systems while enabling multifrequency tuning. Others have proposed similar systems. Ye et al. studied cast-in situ hollow floor slabs equipped with an internal tuned liquid damper, augmenting the structural damping ratio by approximately 2\% [14]. Matia and Gat investigated an elastic beam embedded with a fluid-filled parallel-channel network, and simulation results demonstrated that the fluid-solid interaction reduced structural motion [15]. To the best knowledge of the authors, none have studied the integration of TLCD systems within RC shear wall components.

Prior work from the authors in [13] was focused on the development and validation of an analytical model for TLMCD systems. In this paper, the investigation extends to the TLWD system, whereas perforated openings within RC shear walls are used to integrate a TLMCD. Increasing the height and length of perforated openings may increase structural damping by increasing the size of the internal TLMCD, and it may also significantly reduce the strength capacity and lateral motion performance of the RC shear wall, yet with less significant adverse effects on ultimate flexural capacity [16-18]. Here, both strength and mitigation functionalities are investigated within given geometric constraints to satisfy both strength and motion performance requirements, as well as the trade-off between strength and energy dissipation is studied.

The rest of the paper is organized as follows. Section 2 describes the analytical and numerical models used in conducting the study. This includes the proposed simplified strength model. Section 3 conducts parametric studies for a simplified structure equipped with a TLWD. Section 4 validates the proposed strength model and, further, the parametric evaluation by conducting additional simulation using the validated model. Results from both Sections 3 and 4 are used to establish design considerations for implementing a TLWD system. Section 5 verifies the findings by demonstrating the proposed TLWD on a 42-story building. Section 6 concludes the paper.

\section{Models}

This section presents the models used in conducting the numerical study. It starts by a presentation of the TLMCD analytical model developed in prior work [13], followed by a simplified analytical model to evaluate strength of a TLWD. After that the finite element modeling methodology is described, followed by that of the numerical simulations in MATLAB.

2.1. TLMCD Model. A TLMCD consists of multiple vertical columns jointed with a horizontal tube equipped with multiple orifices located in between adjacent vertical columns. Figure 1(a) is the schematic of a TLMCD and Figure 1(b) illustrates a TLWD system. In Figure 1(a), $\ddot{x}_{g}$ is the acceleration transmitted from the floor, $x_{i}$ is the liquid surface displacement in the ith column, $A$ is the cross-section area of vertical column, $h$ is the initial vertical liquid surface height (equal for every column), $l_{i}$ is the horizontal center-to-center distance between the $i$ th and $i+1$ th columns, $v$ is cross-section area ratio of vertical column to the horizontal tube, and $\psi_{i}$ is the $i$ th orifice blocking ratio.

A nonlinear dynamic model for the TLMCD can be derived using Lagrange equations [13]. Starting with

$$
\frac{\mathrm{d}}{\mathrm{d} t}\left(\frac{\partial T}{\partial \dot{x}_{i}}\right)-\frac{\partial T}{\partial x_{i}}+\frac{\partial V}{\partial x_{i}}=Q_{o i}+Q_{f i}+Q_{e i}, \quad i=1,2, \ldots, N-1,
$$

where $\dot{x}_{i}$ is the liquid velocity in the $i$ th column, $t$ is time, and $Q_{o i}, Q_{f i}$, and $Q_{e i}$ are orifice damping force, friction force between the liquid and inner surface of columns, and liquid inertia force, respectively, the kinematic energy $T$ and potential energy $V$ are taken as

$$
\begin{aligned}
& V=\frac{1}{2} \rho_{l} A g \sum_{i=1}^{N} x_{i}^{2}, \\
& T=\frac{1}{2} \rho_{l} A \sum_{i=1}^{N} \dot{x}_{i}^{2}\left(h+x_{i}\right)+\frac{1}{2} \frac{\rho_{l} A}{v} \sum_{i=1}^{N-1} v^{2} l_{i}\left(\sum_{j=1}^{i} \dot{x}_{j}\right)^{2},
\end{aligned}
$$

where $\rho_{l}$ is the liquid density. The analytical model is based on the following assumptions: (1) all vertical columns are identical in geometry, (2) liquid compression and liquid-air interface diffusion are negligible, resulting in a constant total liquid volume during vibrations, and (3) the characteristic dimensions of the columns' cross sections are considerably smaller than the total length of the tube, resulting in uniform motion of the liquid surface over the cross sections. An $N$ column TLMCD can be treated as $N-1$ degree of freedoms (DOFs) by allowing the displacement of the last column be dependent on the other columns' displacements using the assumed kinematic constraint $x_{N}=-\sum_{i=1}^{N-1} x_{i}$. It should be noted that the analytical model becomes that of a conventional TLCD for $N=2$. The damping force $Q_{o i}$ is assumed to be proportional to the square of the liquid velocity $\dot{x}_{l}^{2}$ [9]. The energy dissipated by the orifice damping force $W_{o}$ can be written as

$$
\delta W_{o}=-\sum_{k=1}^{N-1} \frac{1}{2} \rho_{l} A\left[v \eta_{k}\left|\sum_{j=1}^{k} \dot{x}_{j}\right|\left(\sum_{j=1}^{k} \dot{x}_{j}\right) \sum_{j=1}^{k} \delta x_{j}\right]
$$




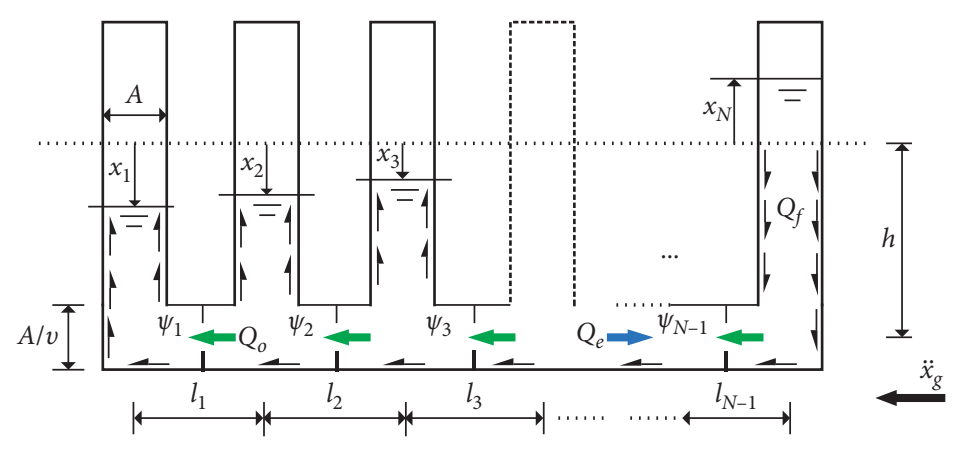

(a)

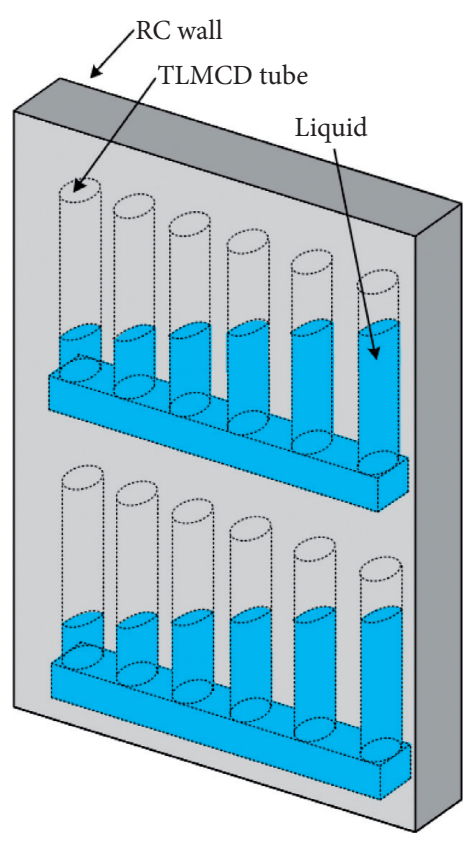

(b)

FIgURE 1: Schematic of (a) TLMCD [13] and (b) TLWD system consisting of a shear wall with embedded TLMCDs.

$Q_{o i}$ on the $i$ th DOF and selected head loss coefficient model $\eta$ are written as [19]

$$
\begin{aligned}
Q_{o i} & =\frac{\partial W_{o}}{\partial x_{i}}=-\frac{1}{2} \sum_{k=i}^{N-1} \rho_{l} A\left[v \eta_{k}\left|\sum_{j=1}^{k} \dot{x}_{j}\right|\left(\sum_{j=1}^{k} \dot{x}_{j}\right)\right], \\
\eta & =\left(\psi+0.707 \psi^{0.375}\right)^{2}(1-\psi)^{-2} .
\end{aligned}
$$

In addition to the head loss effect caused by the orifice blocks, friction resistance between the liquid and inner tube surface provokes a friction head loss effect. For mathematical tractability, the friction force is assumed to be proportional to the liquid velocity $\dot{x}_{l}^{2}$. An expression for the energy dissipated by the friction force $W_{f}$ can be written as

$$
\delta W_{f}=-\sum_{k=1}^{N} \rho_{l} A\left[\mu\left|\dot{x}_{k}\right| \dot{x}_{k}\left(h+x_{k}\right) \delta x_{k}\right]+\sum_{k=1}^{N-1} \rho_{l} A\left[\mu \nu l_{k}\left|\sum_{j=1}^{k} \dot{x}_{j}\right|\left(\sum_{j=1}^{k} \dot{x}_{j}\right) \sum_{j=1}^{k} \delta x_{j}\right]
$$

where $\mu$ is the head loss coefficient due to the friction resistance. The mathematical expression for the friction force $Q_{f i}$ over a small unit of time for the $i$ th DOF is expressed as

$$
Q_{f i}=\frac{\partial W_{f}}{\partial x_{i}}=-\rho_{l} A \mu\left\{\left|\dot{x}_{i}\right| \dot{x}_{i}\left(h+x_{i}\right)+\left|\sum_{j=1}^{N-1} \dot{x}_{j}\right|\left(\sum_{j=1}^{N-1} \dot{x}_{j}\right)\left(h-\sum_{j=1}^{N-1} x_{j}\right)+\sum_{k=i}^{N-1}\left[v l_{k}\left|\sum_{j=1}^{k} \dot{x}_{j}\right|\left(\sum_{j=1}^{k} \dot{x}_{j}\right)\right]\right\} .
$$

The inertial force $Q_{e i}$ is induced by the lateral motion of the building due to an external excitation. Since the liquid mass remains constant during vibrations, the inertial force $Q_{e i}$ on the $i$ th DOF is solely given by the contribution from the liquid motion in the horizontal tube. The energy dissipated by inertial force $W_{e}$ can be written as

$$
\begin{gathered}
\delta W_{e}=-\rho_{l} A \sum_{j=1}^{N-1}\left(\ddot{x}_{g} l_{k} \sum_{j=1}^{k} \delta x_{j}\right), \\
Q_{e i}=\frac{\delta W_{e}}{\partial x_{i}}=-\rho_{l} A \ddot{x}_{g}(t) \sum_{k=i}^{N-1} l_{k} .
\end{gathered}
$$


2.2. TLWD Strength Model. An analytical strength model for the TLWD is developed enabling fast numerical simulations of MDOF representations. The shear wall strength equations from ACI 318-14 [20] are used to calculate the strength of TLWD with a given TLMCD embedment profile. The strength model is built upon considering only the void caused by the vertical columns. A special reinforcement scheme would be required to cope with the void caused by the horizontal tube, which is left to future work. Lateral stiffness $k$, nominal shear strength $V_{n}$, nominal flexural strength $M_{n}$, and nominal axial strength $P_{n}$ are considered as strength objectives. The moment of inertia of TLWD along the in-plane direction can be written

$$
I_{\mathrm{TLWD}}=\frac{1}{12} b_{w} l_{w}^{3}-\sum_{i=1}^{n} A_{i} \bar{y}_{i}^{2}-\frac{1}{4} N \pi r^{4},
$$

where $b_{w}$ is the width of the wall section, $l_{w}$ is the wall length, $A_{i}$ is the area of $i$ th vertical column, $\bar{y}_{i}$ is the distance of the neutral axis to the center of $i$ th circular opening, $N$ is the number of vertical columns, and $r$ is the radius of openings. Stiffness value $k$ for the shear wall with respect to the section installed above is inversely proportional to its deflection when subjected to a unit point load applied at the top. The total deflection of the TLWD is determined from the sum of shear deflection $\Delta_{v}$ and moment deflection $\Delta_{m}$, with $k$ defined as [21]

$$
k=\frac{1}{\Delta_{m}+\Delta_{v}} .
$$

Quantities $\Delta_{v}$ and $\Delta_{m}$ for a TLWD subjected to a unit horizontal load $F$ can be calculated as

$$
\Delta_{m}= \begin{cases}\frac{F h_{w}^{3}}{12 E_{c} I_{\mathrm{TLWD}}}, & \text { for a fixed wall } \\ \frac{F h_{w}^{3}}{3 E_{c} I_{\mathrm{TLWD}}}, & \text { for a cantilever wall }\end{cases}
$$

for a cantilever wall,

$$
\begin{aligned}
\Delta_{v} & =\frac{1.2 F h_{w}}{A_{w} G}, \\
E_{c} & =4700 \sqrt{f_{c}^{\prime}}, \\
G & =\frac{E_{c}}{2(1+v)},
\end{aligned}
$$

where $h_{w}$ is height of wall, $E_{c}$ is Young's modulus, $G$ is shear modulus, $v$ is Poisson's ratio, $f_{c}^{\prime}$ is compressive strength, and $A_{w}=A_{t}-N A$ is the cross-section area of the TLWD taken by removing the cross-section area of the vertical columns $N A$ from the cross-section area of the wall geometry $A_{t}$. The shear strength $V_{n}$ of a TLWD consists of the summation of the shear strength from concrete $V_{c}$ and that from transverse reinforcements $V_{s}$ :

$$
V_{n}=V_{c}+V_{s}
$$

Shear strength values are modified based on the simplified method from ACI-318 Section 11.5.4.5 using the reduction factor $\alpha$ :

$$
\alpha=\frac{I_{\mathrm{TLWD}}}{\left(b_{w} l_{w}^{3} / 12\right)},
$$

with the expressions for $V_{c}$ and $V_{s}$ written as

$$
\begin{aligned}
& V_{c}=0.166 \alpha \sqrt{f_{c}^{\prime}}\left(b_{w} d-N A\right), \\
& V_{s}=\frac{\alpha A_{v} f_{y} d}{s},
\end{aligned}
$$

where $d$ is the effective depth of the cross section, taken as 0.8 $l_{w}$ from the strain compatibility analysis [20]. $A_{v}$ is the crosssectional area of transverse reinforcement, $f_{y}$ is the yield strength of the steel reinforcement, and $s$ is the center-tocenter spacing of transverse reinforcement. Value $P_{n}$ is governed by two failure cases, namely, crushing of concrete and buckling of steel reinforcement. From ACI 318-14, $P_{n}$ is taken as the sum of axial load capacity of concrete and steel reinforcement, with

$$
P_{n}=0.85 f_{c}^{\prime}\left(A_{t}-N A-A_{s}\right)+f_{y} A_{s},
$$

where $A_{s}$ is the cross-sectional area of longitudinal reinforcement. Both the effects of axial load and bending moment are considered in the axial-bending interaction $M_{n}$ illustrated for an 8-column TLWD in Figure 2, assuming an equivalent rectangular compressive stress distribution for the concrete and an elasto-plastic constitutive law for steel reinforcement.

In Figure $2, \varepsilon_{c u}$ is the ultimate compressive strain of concrete, $\varepsilon_{s}$ is the strain of steel reinforcement, $\varepsilon_{c}$ is the strain of concrete, $\varepsilon_{y}$ is the yield strain of steel reinforcement, $\beta$ is the ratio of the depth of the rectangular stress block to the neutral axis, $C_{c}$ is the compression force from concrete, $C_{s}$ is the compression force from steel reinforcement, and $T_{s}$ is the tension force from steel reinforcement. The value for $M_{n}$ given an applied axial load $N_{u}$ can be calculated using equations (17)-(21) based on the strain distribution under force equilibrium:

$$
\begin{aligned}
M_{n} & =\sum_{i=1} A_{s}^{i} \varepsilon_{c}^{i} E_{s}\left(c-d_{c}^{i}\right)+C_{c}(c / 2)+\sum_{i=1} A_{s}^{i} \varepsilon_{t}^{i} E_{s}\left(d_{t}^{i}-c\right) \\
C_{s} & =\sum_{i=1} A_{s}^{i} \varepsilon_{c}^{i} E_{s} \\
T_{s} & =\sum_{i=1} A_{s}^{i} \varepsilon_{t}^{i} E_{s} \\
C_{c} & =0.85 f_{c}^{\prime} \beta A_{c b} \\
C_{s} & +C_{c}=T_{s}+N_{u}
\end{aligned}
$$

where $\varepsilon_{c}^{i}$ and $\varepsilon_{s}^{i}$ are, respectively, the compressive and tensile strain of the reinforcement bar at the ith depth with $\varepsilon_{s} \leq \varepsilon_{y}$, 


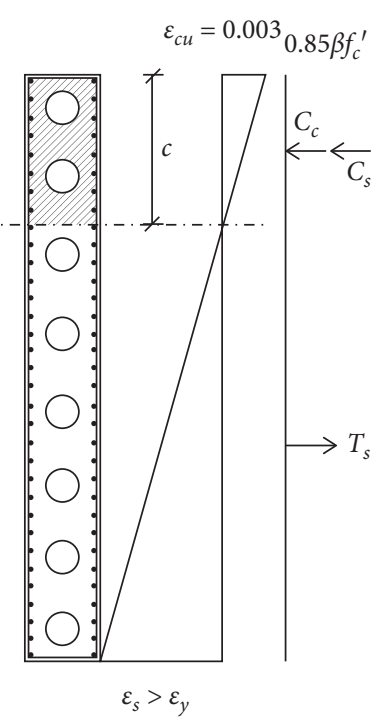

(a)

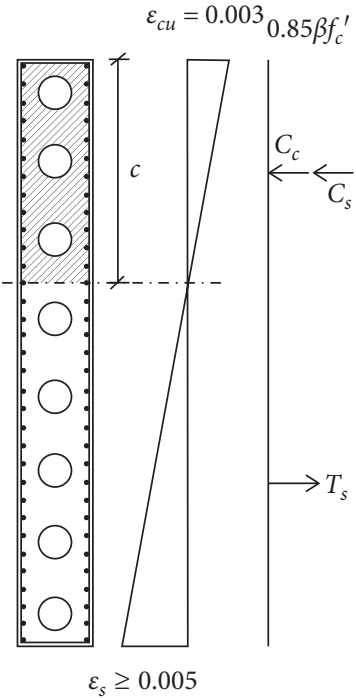

(b)

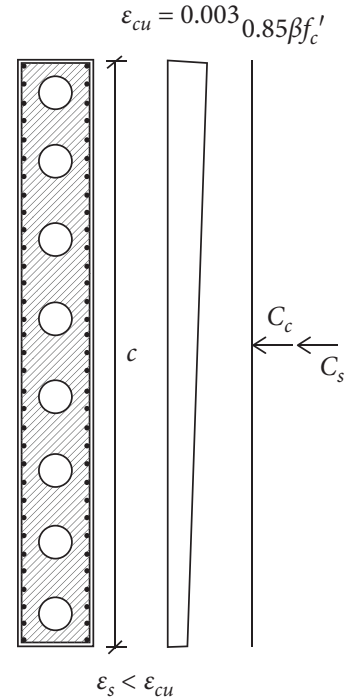

(c)

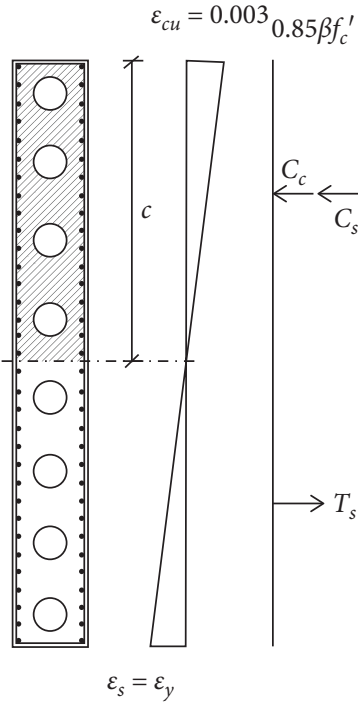

(d)

FiguRE 2: Strain distribution and resultant forces for an 8-column TLWD under (a) pure bending, (b) tension-controlled, (c) compressioncontrolled, and (d) balanced compression and tension.

$d_{c}^{i}$ and $d_{s}^{i}$ are, respectively, the depth of the $i$ th reinforcement bar in compression and tension, $E_{s}$ is the Young modulus of steel, $A_{s}^{i}$ is the cross-section area of reinforcement bar at the $i$ th depth, and $A_{c b}$ is the area of compression block.

This TLWD strength model will be validated in Section 4 using data produced by numerical simulations.

2.3. Finite Element Model. The finite element model of a TLWD is constructed to validate the TLWD strength model. The concrete damage plasticity model (CDPM) in ABAQUS is used to simulate the nominal strength of TLWDs. The CDPM considers both tensile cracking and compressive crushing failure mechanisms based on the models proposed by Lubliner et al. [22] and Lee and Fevens [23]. In the CDPM, the five following parameters characterize the stressstrain behavior and damage of inelastic and cracking strain. The dilation angle $\psi$ defines the angle of the inclination surface towards the hydrostatic axis. The eccentricity $\varepsilon$ defines the shape of the hyperbola and can be calculated as the ratio of tensile strength to compressive strength [24]. Parameter $f_{b 0} / f_{c 0}$ is the ratio of compressive strength in biaxial direction $f_{b 0}$ to uniaxial direction $f_{c 0}$, recommended as 1.16 in [25]. Parameter $K_{c}$ defines the shape of the cross section at failure in the deviatoric plane, recommended as 0.67 in [25]. The viscosity parameter $\mu$ is used to reduce the time interval to prevent divergence. For nonviscoelastic materials such as concrete, the value should be as small as possible [24]. Parameters used in characterizing the CDPM are listed in Table 1.

The uniaxial compressive stress-strain relationship is characterized following Hsu and Hsu model [26]. The stressstrain relationship, plotted in Figure 3(a), solely relies on $f_{c}^{\prime}$, with
TABLE 1: Concrete damage plasticity model parameters.

\begin{tabular}{lcc}
\hline Parameters & Value & Unit \\
\hline$\psi$ & 31 & Degree \\
$\varepsilon$ & 0.1 & \\
$f_{b 0} / f_{c 0}$ & 1.16 & - \\
$K_{c}$ & 0.667 & - \\
$\mu$ & 0.0001 & - \\
\hline
\end{tabular}

$$
\begin{aligned}
\sigma_{c} & =\varepsilon_{c} E_{c}, \quad \text { for } \varepsilon_{c} \leq \varepsilon_{l}, \\
\varepsilon_{l} & =\frac{0.5 f_{c}^{\prime}}{E_{c}}, \\
\sigma_{c} & =f_{c}^{\prime}\left[\frac{\beta\left(\varepsilon_{c} / \varepsilon_{0}\right)}{\beta-1+\left(\varepsilon_{c} / \varepsilon_{0}\right)}\right], \quad \text { for } \varepsilon_{c}>\varepsilon_{l}, \\
\beta & =\frac{1}{1-\left(f_{c}^{\prime} / \varepsilon_{0} E_{c}\right)} \\
\varepsilon_{0} & =1.29 \times 10^{-5} f_{c}^{\prime}+0.00211,
\end{aligned}
$$

where $\sigma_{c}$ is the compressive stress, $\varepsilon_{l}$ is the compressive strain at the end of linear phase, and $\varepsilon_{0}$ is the compressive strain at peak stress.

The tensile stress-strain relationship of concrete is characterized using Nayal and Rasheed tension stiffening model [27] modified by Wahalathantri et al. [28]. The tensile behavior, plotted in Figure 3(b), is linear until reaching the average splitting strength $f_{c t}$, corresponding to the cracking strain $\varepsilon_{\text {cr. }}$. After, the stress decreases at various rates after reaching $0.77 f_{c t}$, corresponding to $1.25 \varepsilon_{c r}, 0.45 f_{c t}$, corresponding to $4 \varepsilon_{c r}$, and $0.1 f_{c t}$, corresponding to $8.7 \varepsilon_{\mathrm{cr}}$, the cracking strain $\varepsilon_{\mathrm{cr}}$ and average splitting strength $f_{c t}$ are written as 

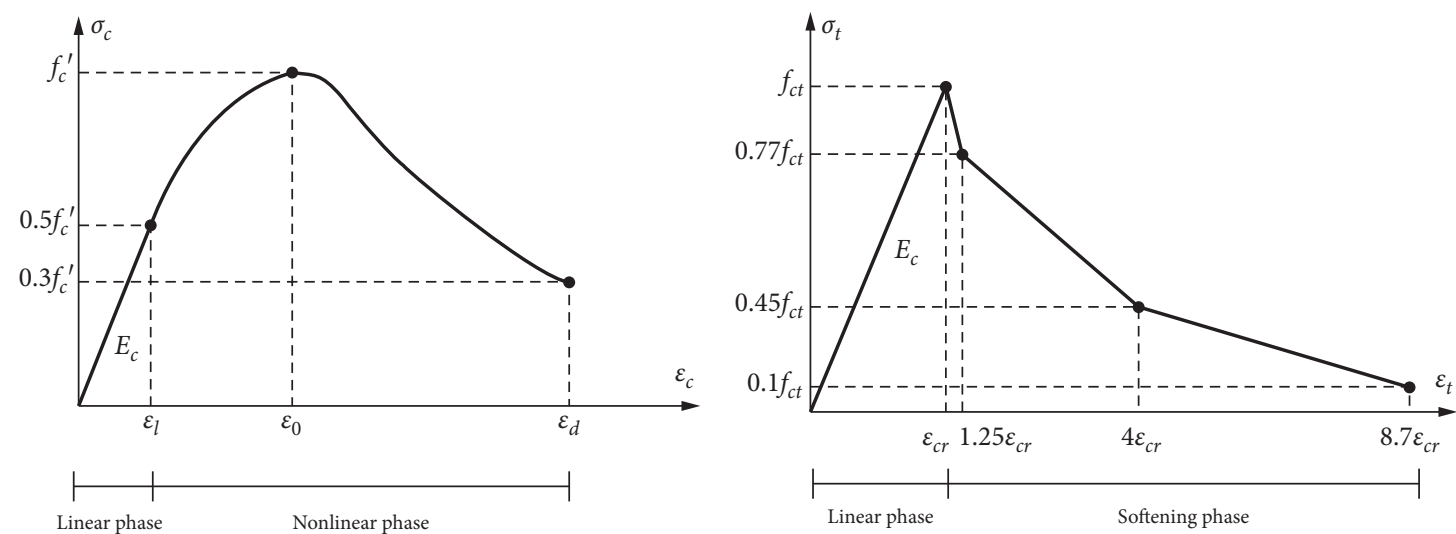

(b)

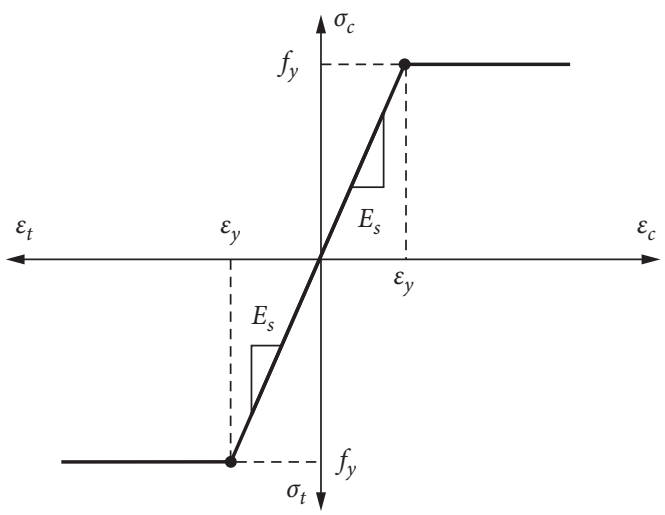

(c)

Figure 3: Materials stress-strain relationship: (a) uniaxial compression of concrete, (b) uniaxial tension of concrete, and (c) bilinear curve of steel.

$$
\begin{aligned}
\varepsilon_{\mathrm{cr}} & =\frac{f_{c t}}{E_{c}}, \\
f_{c t} & =0.56 \sqrt{f_{c}^{\prime}} .
\end{aligned}
$$

The constitutive model of steel reinforcement bars is assumed to be bilinear elastic-perfectly plastic with identical stress-strain behavior in tension and compression, as shown in Figure 3(c). $f_{y}, \varepsilon_{y}$, and $E_{s}$ are the yield strength, yield strain, and Young's modulus of steel reinforcement accordingly. The bond between reinforcement steel and concrete is assumed to be perfect:

$$
\begin{array}{ll}
\sigma_{t}=E_{s} \varepsilon_{t}, & \text { for } \varepsilon_{t} \leq \varepsilon_{y}, \\
\sigma_{t}=f_{y}, & \text { for } \varepsilon_{t}>\varepsilon_{y} .
\end{array}
$$

Concrete and steel reinforcement are modeled using linear 3D 8-node solid and linear 2D 2-node truss elements. The embedded region constraint method is employed, where concrete and steel reinforcement is modeled as host and embedded regions, respectively. The embedded TLMCDs are modeled as vertical voids occupied by liquid. A mesh sensitivity study is conducted to achieve convergence. The element size of concrete is determined as $50 \times 80 \times 110 \mathrm{~mm}^{3}$ (width $\times$ length $\times$ height) and the meshing length of steel as $150 \mathrm{~mm}$, with a total of approximately 85,000 elements.

2.4. Numerical Simulation Model. After the TLWD validation using the finite element model, the TLWD will be numerically simulated in the MATLAB environment. The equation of motion for a structure equipped with a TLWD can be written as

$$
\mathbf{M} \ddot{\mathbf{x}}+\mathbf{C} \dot{\mathbf{x}}+\mathbf{K x}=\mathbf{E}_{d} \mathbf{F}_{d}+\mathbf{E}_{e} \mathbf{F}_{e},
$$

where $\mathbf{M}, \mathbf{C}$, and $\mathbf{K}$ are the mass, damping, and stiffness system matrices, respectively, $\mathbf{x}$ is the displacement vector, the dot denotes a time derivative, $\mathbf{F}_{d}$ is the damping forces generated by TLWD, $\mathbf{F}_{e}$ is the excitation force vector, and $\mathbf{E}_{d}$ and $\mathbf{E}_{e}$ are the associated force location matrices. The statespace formulation of equation (25) is written as 


$$
\begin{aligned}
\dot{\mathbf{X}} & =\mathbf{A} \mathbf{X}+\mathbf{B}_{d} \mathbf{F}_{d}+\mathbf{B}_{e} \mathbf{F}_{e}, \\
\mathbf{X} & =\left[\begin{array}{l}
\mathbf{x} \\
\dot{\mathbf{x}}
\end{array}\right], \\
\mathbf{A} & =\left[\begin{array}{cc}
\mathbf{0} & \mathbf{I} \\
-\mathbf{M}^{-1} \mathbf{K} & -\mathbf{M}^{-1} \mathbf{C}
\end{array}\right], \\
\mathbf{B}_{d} & =\left[\begin{array}{c}
\mathbf{0} \\
\mathbf{M}^{-1} \mathbf{E}_{d}
\end{array}\right], \\
\mathbf{B}_{e} & =\left[\begin{array}{c}
\mathbf{0} \\
\mathbf{M}^{-1} \mathbf{E}_{e}
\end{array}\right],
\end{aligned}
$$

where $\mathbf{X}$ is the state vector, $\mathbf{A}$ is the state matrix, and $\mathbf{B}_{d}$ and $\mathbf{B}_{e}$ are the input vector. From [13], an $N$-column symmetric TLWD can be simplified into a linear system having N/2 equal pairwise natural frequencies and can be tuned through adjusting column spacings. The system stiffness matrix $\mathbf{K} \epsilon$ $\mathbb{R}^{((N / 2)+1) \times((N / 2)+1)}$, mass matrix $\mathbf{M} \in \mathbb{R}^{((N / 2)+1) \times((N / 2)+1)}$, and damping matrix $\mathbf{C} \in \mathbb{R}^{((N / 2)+1) \times((N / 2)+1)}$ of the coupled SDOF structure can be written as

$$
\mathbf{K}=\left[\begin{array}{ccccc}
k_{s} & 0 & 0 & \ldots & 0 \\
0 & 2 \rho A g & 0 & \ldots & 0 \\
0 & 0 & 2 \rho A g & \ldots & 0 \\
\vdots & \vdots & \vdots & \ddots & 0 \\
0 & 0 & 0 & \ldots & 2 \rho A g
\end{array}\right]
$$

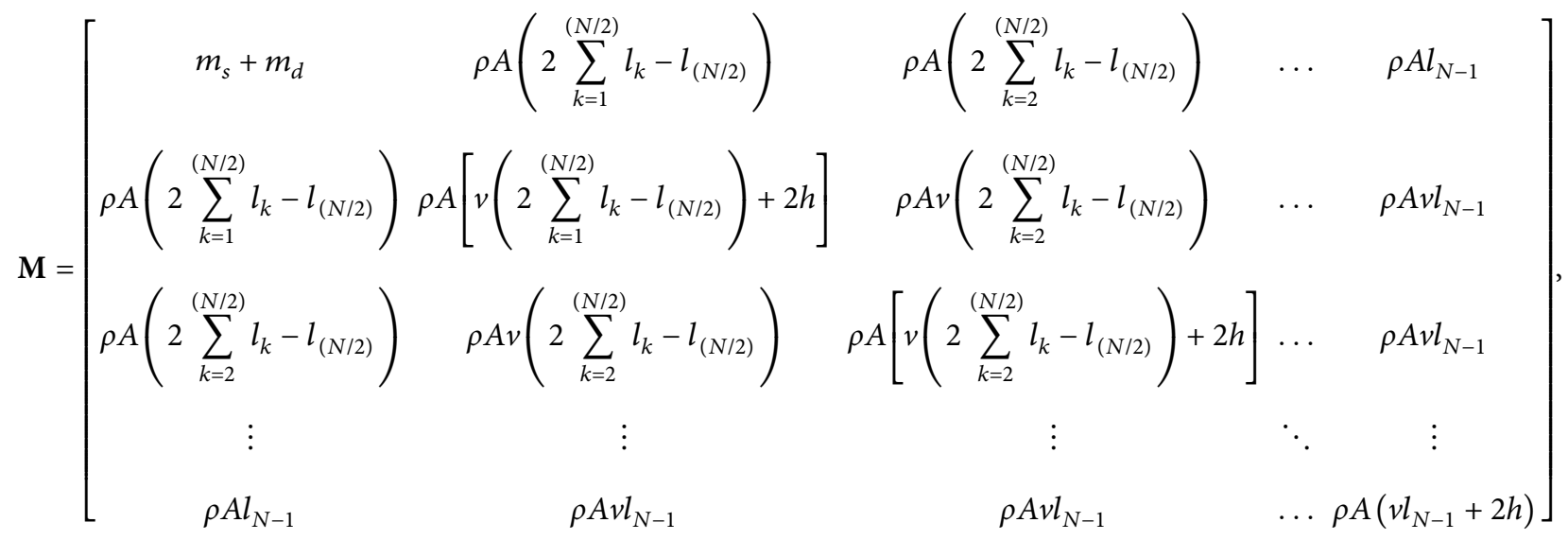

$$
m_{d}=\rho l A\left(N h+\sum_{k=1}^{N-1} \frac{l_{k}}{v}\right),
$$

$$
\mathbf{C}=\left[\begin{array}{cccc}
c_{s} & 0 & \ldots & 0 \\
0 & 0 & \ldots & 0 \\
\vdots & \vdots & \ddots & 0 \\
0 & 0 & \ldots & 0
\end{array}\right],
$$

where $m_{d}$ is the liquid mass of the TLMCD and $c_{s}$ is the structural damping coefficients. It should be noted that the linear damping coefficients of TLMCDs are taken equal to 0 in the linear damping matrix C because the TLMCD damping force is proportional to the square of velocity (equations (4) and (7)). The state vector $\mathbf{X}=\left[\begin{array}{ll}\mathbf{x} & \dot{\mathbf{x}}\end{array}\right]^{T} \epsilon$ 
$\mathbb{R}^{(N+2)}$ is defined for the coupled SDOF system. An explicit discrete-time formulation is used to solve the state-space equation:

$$
\mathbf{X}\left(t+\Delta_{t}\right)=e^{\mathbf{A} \Delta t} \mathbf{X}(t)+\mathbf{A}^{-1}\left(e^{\mathbf{A} \Delta t}-\mathbf{I}\right)\left[\mathbf{B}_{e} \mathbf{F}_{e}(t)+\mathbf{B}_{d} \mathbf{F}_{d}(t)\right],
$$

where $e$ is the exponential function and I the identity matrix.

\section{Performance Investigation: Single Wall Section}

In this section, the motion and strength performance of the TLWD are preliminarily investigated on a simplified SDOF system and a single RC wall geometry, respectively. The investigation starts by a parametric study on the TLMCD configuration for motion engineering. It is followed by an investigation of embedment strategies to optimize performance versus both motion and strength by comparing the numerical and FEM results. After that, results from the finite element analysis are used to validate the simplified analytical model for strength. Then, the validated model is used to further the study on trade-offs between motion and strength performance.

The SDOF system is based on a representation of a $42-$ story residential building (Building 1A) [29] equipped with a TLWD. Note that the behavior of the SDOF system does not fully represent that of the multi-degree-of-freedom system (MDOF). The structural mass $m_{s}$ and fundamental frequency $\omega_{\mathrm{s}}$ are obtained from the literature [29]. The structural damping coefficients are obtained assuming 5\% natural damping ratio. The SDOF system is schematized in Figure 4 and model parameters are summarized in Table 2. The vertical liquid columns in the attached TLWD are equally spaced, and the first vibration mode is tuned to the fundamental frequency of the structure. The TLWD represents the assembly of numerous TLMCDs occupying an equivalent cross-section $A_{\mathrm{eq}}$.

The strength evaluation assumes the TLMCDs are embedded in an interstory wall at the ground level from Building 1A [29]. The wall, illustrated in Figure 5, has a height $h_{w}=4,167 \mathrm{~mm}$, length $l_{w}=5,334 \mathrm{~mm}$, and width $b_{w}$ $=610 \mathrm{~mm}$. It is reinforced using $1.39 \%$ longitudinal and $0.58 \%$ transverse reinforcement ratio. The concrete compressive strength $f_{c}^{\prime}$ and steel reinforcement yield strength $f_{y}$ are taken as $55.16 \mathrm{MPa}$ and $413.69 \mathrm{MPa}$, respectively.

Transfer functions $H_{1}(\omega)$ and $H_{2}(\omega)$ are selected as the main motion performance objectives. They correspond to the amplification of displacement and acceleration responses in the frequency domain, respectively. They are evaluated numerically from the steady-state response using

$$
\begin{aligned}
& H_{1}(\omega)=\frac{\left|x_{s}(\omega)\right|_{\max }}{\left(p_{0} / k_{s}\right)}, \\
& H_{2}(\omega)=\frac{\left|\ddot{x}_{s}(\omega)\right|_{\max }}{\left(p_{0} / m_{s}\right)} .
\end{aligned}
$$

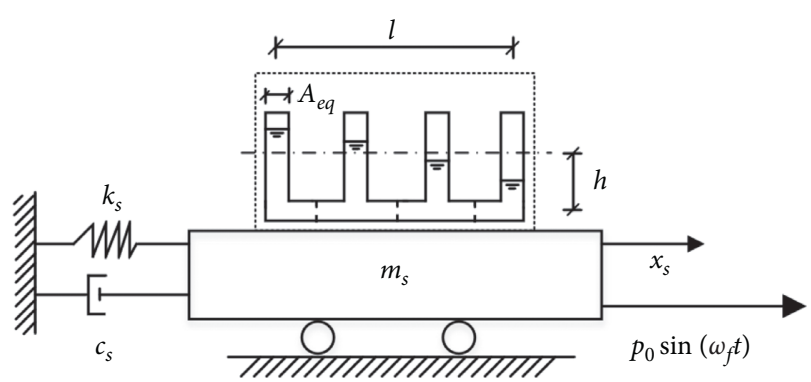

FIgURE 4: Schematic of the SDOF system equipped with a TLWD.

TABle 2: Properties of the SDOF system.

\begin{tabular}{lccc}
\hline Parameter & Variable & Value & Unit \\
\hline Structural mass & $m_{s}$ & 38,540 & Tons \\
Structural frequency & $\omega_{\mathrm{s}}$ & 1.57 & $\mathrm{rad} / \mathrm{s}$ \\
Structural stiffness & $k_{s}$ & 95,094 & $\mathrm{kN} / \mathrm{m}$ \\
Structural damping coefficients & $c_{s}$ & 6,054 & $\mathrm{kN} \cdot \mathrm{s} / \mathrm{m}$ \\
TLMCD length & $l$ & 5,050 & $\mathrm{~mm}$ \\
TLMCD liquid height & $h$ & 600 & $\mathrm{~mm}$ \\
Excitation magnitude & $p_{0}$ & 378 & $\mathrm{kN}$ \\
\hline
\end{tabular}

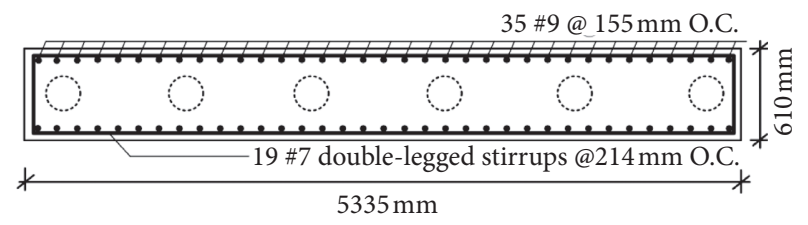

FIGURE 5: Cross-section view of the RC wall and embedded location of a single-layered 6-column TLMCD (columns in dashed line).

Nominal shear $V_{n}$, flexural $M_{n}$, and axial strength $P_{n}$ are selected as the main strength objectives, simulated through the displacement-controlled pushover analysis at the ultimate stage. For the same wall cross section, slender wall and short wall geometries are used to reach the flexural and shear failure modes separately. Among these, $M_{n}$ is determined based on balanced axial-moment interaction from strain compatibility analysis with approximated axial load ratio $\rho_{\text {axial }}=0.36$.

3.1. Motion Performance. The performance of the TLMCD is first investigated on single-layered configurations (i.e., all columns organized along the same horizontal line in Figure 5), with the overall geometry constrained to the shear wall's geometry. The parametric studies on the number of columns $N$ and their diameter $D$ are conducted with $D$ expressed as a function of wall thickness $b_{w}$. A harmonic excitation of frequency ranging from $0.8 \omega_{\mathrm{s}}$ to $1.2 \omega_{\mathrm{s}}$ is used. Tuning frequency ratio $f$ and head loss coefficient $\eta$ are adjusted to obtain the optimal transfer functions $\left.H_{1}(\omega)\right|_{\text {opt }}$ and $\left.H_{2}(\omega)\right|_{\text {opt }}$. Both $\left.H_{1}(\omega)\right|_{\text {opt }}$ and $\left.H_{2}(\omega)\right|_{\text {opt }}$ over various values for $D$ and $N$ are plotted in Figures 6 and 7, respectively. Tables 3 and 4 list the corresponding mass ratio $\bar{m}$ of the equivalent TLMCD to the structural mass, the optimal tuning frequency $\left.f\right|_{\text {opt }}$, the optimal head loss coefficient $\left.\eta\right|_{\text {opt }}$ 


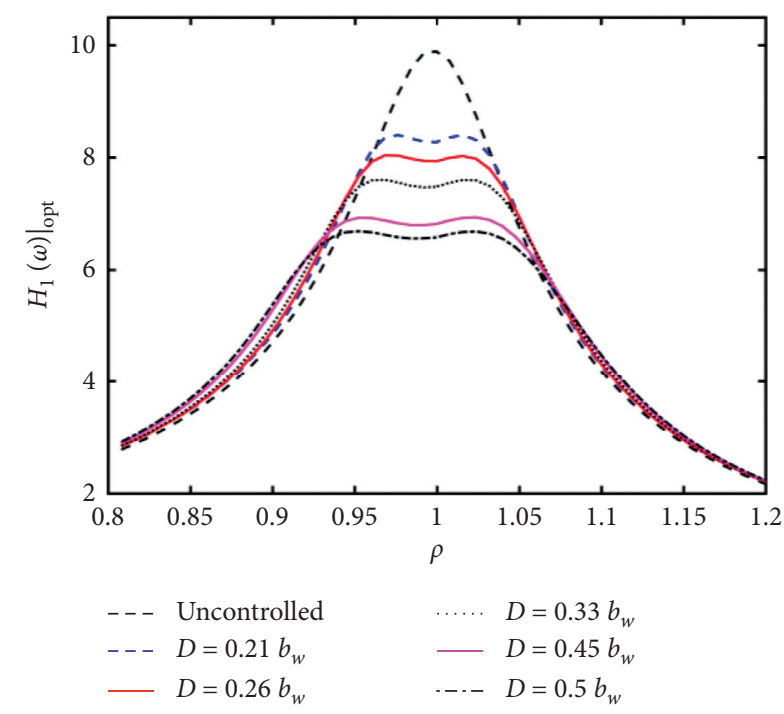

(a)

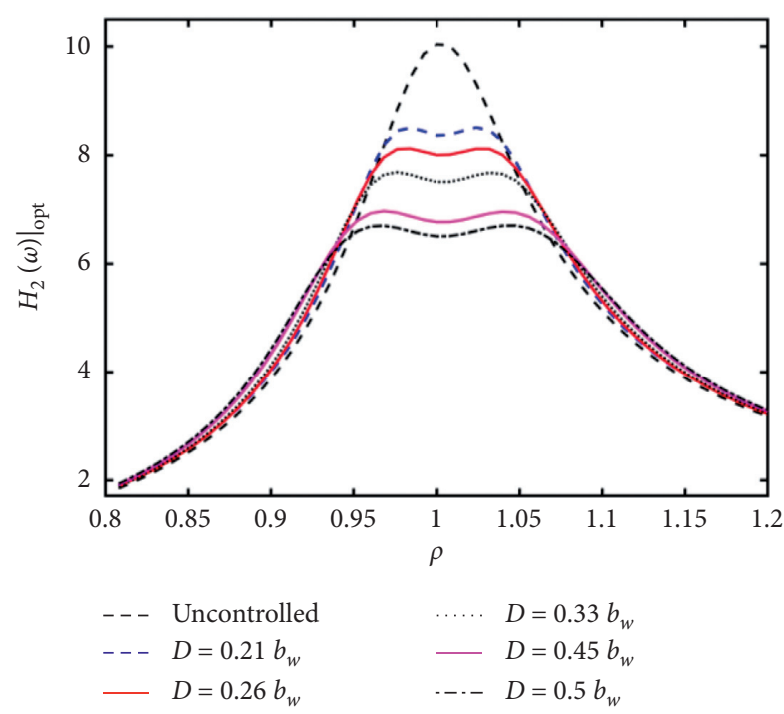

(b)

FIGURE 6: Transfer functions plot of 6-vertical column TLMCDs with various column diameter: (a) transfer function of displacement and (b) transfer function of acceleration.

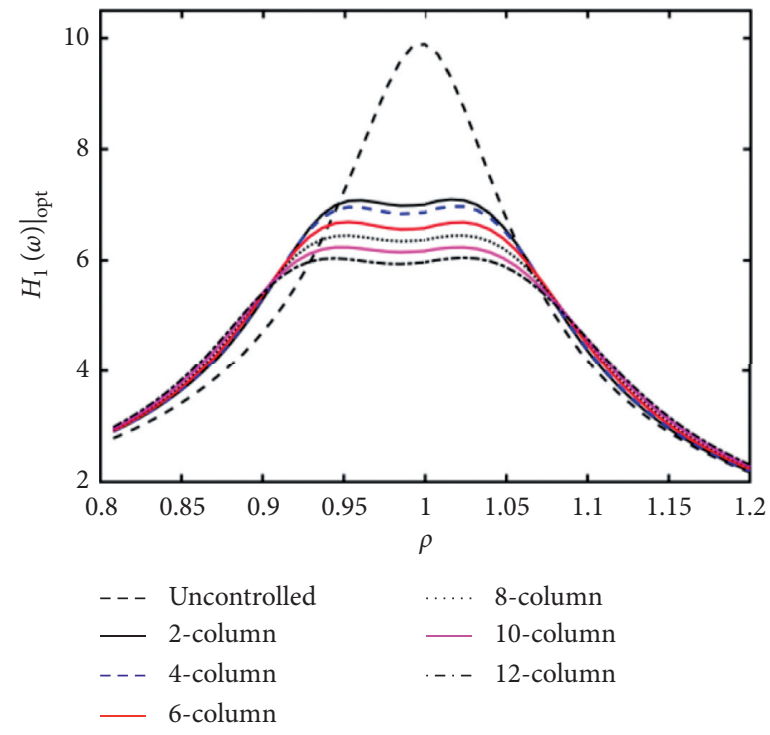

(a)

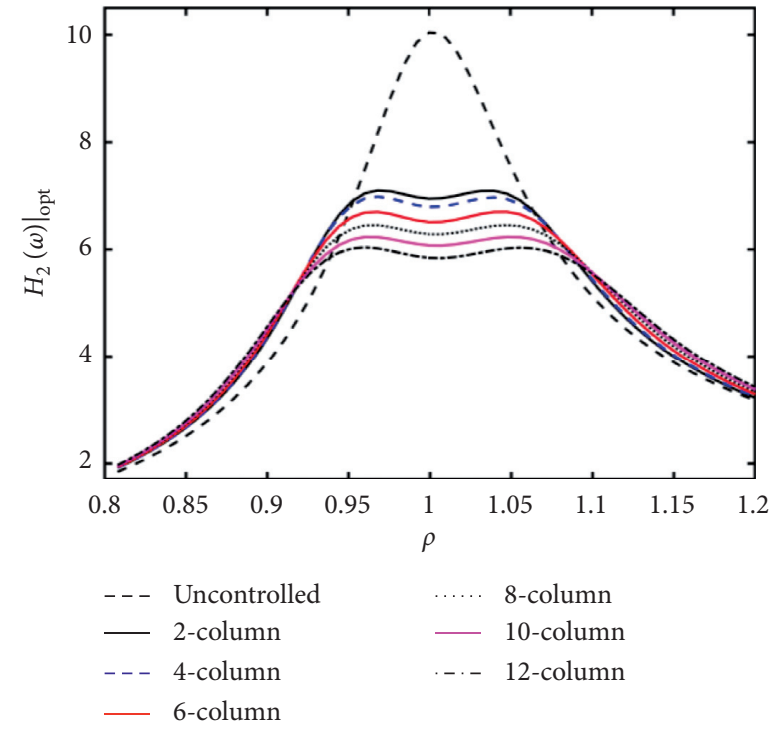

(b)

FIGURE 7: Transfer functions plot of $0.5 b_{w}$ columns' diameter TLMCDs with various number of columns: (a) transfer function of displacement and (b) transfer function of acceleration.

TABLE 3: Optimal 6-column TLWD parameters under various column diameters $D$.

\begin{tabular}{lccccc}
\hline$D$ & $\bar{m}(\%)$ & $\left.f\right|_{\text {opt }}$ & $\left.\eta\right|_{\text {opt }}$ & $W_{d}(K J)$ & 19.97 \\
\\
\hline $0.21 b_{w}$ & 0.23 & 0.993 & 0.28 & 23.38 & 26 \\
$0.26 b_{w}$ & 0.36 & 0.992 & 0.49 & 26.98 & 203 \\
$0.33 b_{w}$ & 0.55 & 0.989 & 0.74 & 31.34 & 139 \\
$0.45 b_{w}$ & 1.04 & 0.984 & 1.41 & 32.36 & 124 \\
$0.5 b_{w}$ & 1.27 & 0.982 & 1.85 & 200 \\
\hline
\end{tabular}


TABLE 4: Optimal $0.5 b_{w}$-column diameter TLWD parameters under various numbers of column $N$.

\begin{tabular}{lccccc}
\hline$N$ & $\bar{m}(\%)$ & $\left.f\right|_{\text {opt }}$ & $\left.\eta\right|_{\text {opt }}$ & $W_{d}(\mathrm{~kJ})$ & $\left.x_{l}\right|_{\max }(\mathrm{mm})$ \\
\hline 2 & 0.71 & 0.987 & 10.2 & 11.91 & 162 \\
4 & 0.93 & 0.985 & 3.14 & 21.49 & 145 \\
6 & 1.27 & 0.982 & 1.85 & 32.26 & 124 \\
8 & 1.62 & 0.979 & 1.23 & 44.02 & 105 \\
10 & 1.97 & 0.976 & 0.93 & 56.35 & 93 \\
12 & 2.33 & 0.973 & 0.74 & 68.29 & 82 \\
\hline
\end{tabular}

taken constant throughout the TLMCD and the energy dissipated by cycle $W_{d}$, under each $D$ and $N$, respectively.

Results show that increasing the columns' diameters or numbers has a positive effect on mitigation capabilities, as it would be expected due to the increase in liquid mass. The lower column diameter cases reduce the maximum total damping forces but result in a larger liquid displacement $x_{l} \mid$ $\max$ in vertical columns, as shown in Table 3. Similarly, the amplification of energy dissipation is observed from results listed in Table 4 when the column number increases from 2 to 8 . This is mainly because the increased mass in the fundamental mode contributes a higher total damping force.

Overall, results confirm that the mitigation performance of the TLWD can be ameliorated by adding liquid mass, but there is a physical limit in doing so because the increased liquid mass yields a decrease in the concrete cross-section area, which will yield a loss in strength. Section 3.2 studies TLMCD-embedded strategies to optimize performance versus both motion and strength.

3.2. Embedment Strategies. A strength performance investigation is conducted under different embedded TLMCD configurations designed to yield similar mitigation performance under various optimal TLMCD configurations. First, single-layer TLMCD configurations are studied. These configurations, designed to arbitrarily maintain $H_{1} \mid$ max and $\left.H_{2}\right|_{\max }$ constant at 6.7, along with the shear strength $V_{n}$, axial-bending strength $M_{n}$, axial strength $P_{n}$, and stiffness $k$ of the wall obtained from the finite element analysis, are listed in Table 5. Results reveal that achieving mitigation performance through a distributed column strategy increases the shear strength and the axial-bending strength, but decreases the axial strength and lateral stiffness, yet with a marginally decreasing change in variations. Overall, distributing the TLMCD over 12 columns instead of 6 provoked a decrease in the concrete area $A_{c}$ of $1.5 \%$, with $V_{n}$ and $M_{n}$ increasing approximately by $9.7 \%$ and $7.8 \%, P_{n}$ and $k$ decreasing about $3.1 \%$ and $1 \%$.

Next, the effect of distributing inertia through a different number of layers of tubes on the TLWD strength is investigated. This study is done on the layered configurations illustrated in Figure 8 (one-, two-, and three-layer configurations), with the TLMCD configurations designed to maintain a constant total cross-section area across each configuration and by optimizing their design to arbitrarily maintain $\left.H_{1}\right|_{\max }$ and $\left.H_{2}\right|_{\max }$ constant at 7.9.
TABLE 5: TLWD strength performance under single-layer configurations.

\begin{tabular}{lccccccc}
\hline$D$ & $N$ & $\bar{m}(\%)$ & $\left.H_{1}\right|_{\max }$ & $\begin{array}{c}V_{n} \\
\left(10^{3} \mathrm{kN}\right)\end{array}$ & $\begin{array}{c}M_{n} \\
\left(10^{3} \mathrm{kN} \cdot \mathrm{m}\right)\end{array}$ & $\begin{array}{c}P_{n} \\
\left(10^{3} \mathrm{kN}\right)\end{array}$ & $\begin{array}{c}k \\
\left(10^{3} \mathrm{kN} / \mathrm{m}\right)\end{array}$ \\
\hline $0.5 b_{w}$ & 6 & 1.26 & 6.7 & 6.47 & 109.8 & 177.4 & 3,748 \\
$0.45 b_{w}$ & 8 & 1.31 & 6.7 & 6.83 & 114.9 & 175.8 & 3,808 \\
$0.42 b_{w}$ & 10 & 1.37 & 6.7 & 6.98 & 118.3 & 172.7 & 3,709 \\
$0.37 b_{w}$ & 12 & 1.4 & 6.7 & 7.1 & 118.4 & 171.8 & 3,710 \\
\hline
\end{tabular}

Table 6 lists the strength values $V_{n}, M_{n}, P_{n}$, and $k$ under each configuration. Results reveal that while the axial strength and lateral stiffness remains unchanged due to the constant concrete cross-section area, distributing liquid mass amongst additional layers negatively affect both shear and axial-bending strengths, with the axial-bending strength significantly decreasing $(27.46 \%)$ between the one- and three-layer configurations. This can be attributed to the larger area occupied by the tubes over the width of the wall, causing stress concentration at the thinnest concrete cross section around middle section of the wall. This is shown by the FEM investigation in Figure 9. In the numerical analysis, concrete cracks were first observed over the middle sections between layers and then propagated to the outer surface. The failure stage was reached right after the formation of vertical sliding cracks. A similar failure pattern was also observed in the one-layer configuration, but, at higher thresholds, it was consistent with Table 6 .

Lastly, the effect of distributing individual TLMCDs over the in-plane direction on mitigation performance is examined. The investigated embedded geometries have a constant number of columns, but the arrangements are divided into $n$ TLMCDs as illustrated in Figure 10, where the concrete strength is approximated as constant across all configurations (i.e., ignoring changes in the horizontal tube) and properties are optimized to minimize the maximum value of the transfer functions. Table 7 lists the results. The examination shows that the utilization of a single-TLMCD arrangement yields significantly higher mitigation capability, attributed to the longer horizontal distance between the opposite columns. Because the length of the TLMCD is constrained by the size of the shear wall, the tuning frequency is only dependent on the ratio of column area to horizontal tube area $v$. Disjointed TLMCDs have a larger $\left.v\right|_{\text {opt }}$, resulting in a less combined liquid mass.

\section{Design of TLWD}

In this section, results from the performance investigation presented in the previous section are used to validate the analytical TLWD strength model. After, the analytical TLWD strength model is used to further investigate tradeoffs between motion and strength performance when designing a TLWD. Lastly, possible design strategies based on findings are discussed.

4.1. TLWD Strength Model Validation. The analytical model developed in Section 2.2 is validated using FEM data from 


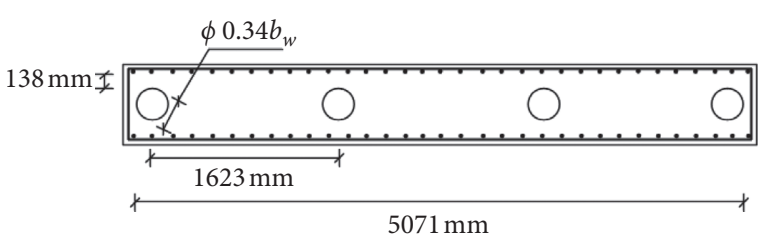

(a)

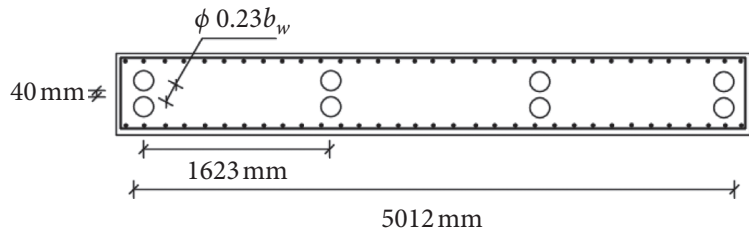

(b)

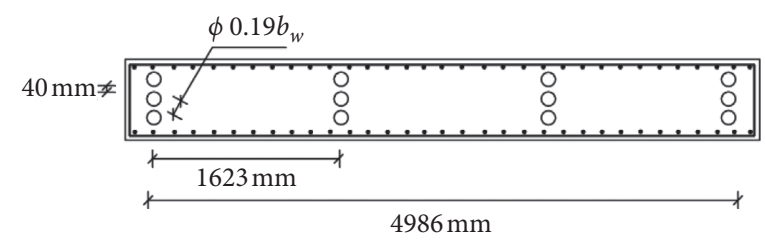

(c)

FIGURE 8: TLWD cross-section view of multiple-layer embedded TLMCD: (a) one-layer 4-column TLWD, (b) two-layer 4-column TLWD, and (c) three-layer 4-column TLWD.

TABLE 6: TLWD strength performance under multiple-layer configurations.

\begin{tabular}{lcccccccc}
\hline No. of layers & $D$ & $N$ & $\bar{m}(\%)$ & $\left.H_{1}\right|_{\max }$ & $V_{n}\left(10^{3} \mathrm{kN}\right)$ & $M_{n}\left(10^{3} \mathrm{kN} \cdot \mathrm{m}\right)$ & $P_{n}\left(10^{3} \mathrm{kN}\right)$ & $K\left(10^{3} \mathrm{kN} / \mathrm{m}\right)$ \\
\hline 1 & $0.34 b_{w}$ & 4 & 0.43 & 7.84 & 8.61 & 134.4 & 198.6 & 4,277 \\
2 & $0.23 b_{w}$ & 4 & 0.43 & 7.84 & 7.95 & 115.5 & 198.8 & 4,255 \\
3 & $0.19 b_{w}$ & 4 & 0.43 & 7.84 & 7.64 & 97.5 & 198.8 & 4,242 \\
\hline
\end{tabular}

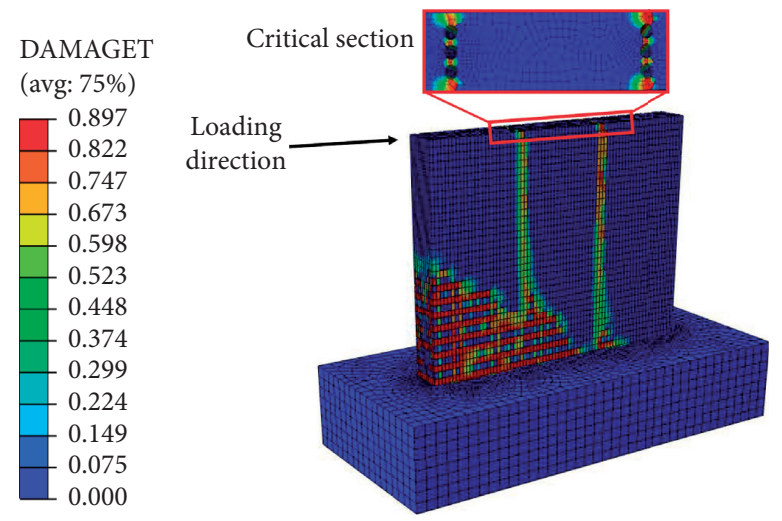

FIGURE 9: Concrete tension damage distribution of TLWD with three-layered embedment profile (DMAGET indicates tension damage of the concrete).

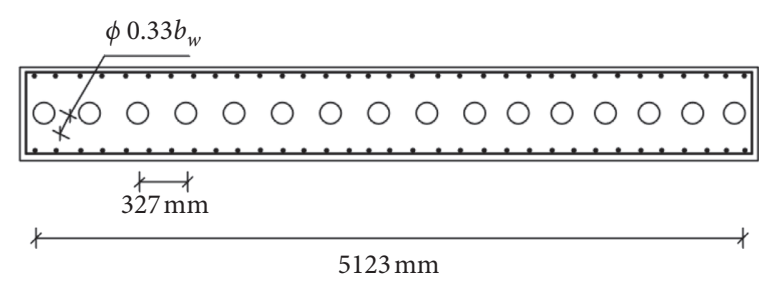

(a)

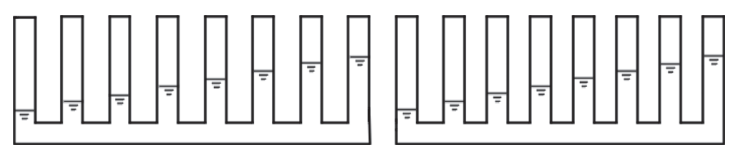

(c)

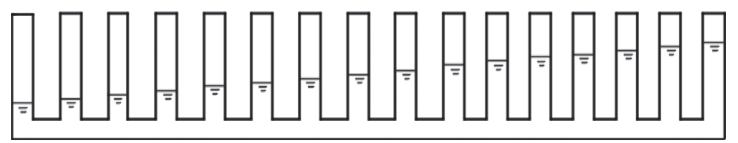

(b)
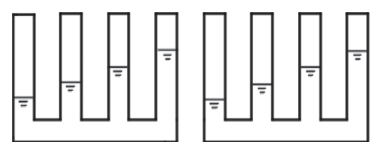

(d)

FIGURE 10: One-layer vertical column arrangement organized into (a) one 16-column TLMCD, (b) two 8-column TLMCDs, and (c) four 4column TLMCDs. 
TABLE 7: Results from distributed TLMCD investigation.

\begin{tabular}{lcccccccc}
\hline$D$ & $n$ & $N$ & $\bar{m}(\%)$ & $\left.f\right|_{\text {opt }}$ & $\left.v\right|_{\text {opt }}$ & $\left.\eta\right|_{\text {opt }}$ & $\left.H_{1}\right|_{\max }$ & $\left.H_{2}\right|_{\max }$ \\
\hline $0.33 b_{w}$ & 1 & 16 & 1.47 & 0.981 & 0.41 & 0.25 & 6.64 & 6.68 \\
$0.33 b_{w}$ & 2 & 8 & 0.8 & 0.989 & 1.42 & 0.28 & 8.04 & 8.07 \\
$0.33 b_{w}$ & 4 & 4 & 0.62 & 0.993 & 4.66 & 0.28 & 8.94 \\
\hline
\end{tabular}

TABLE 8: Structural strengths obtained by the FEM and analytical model.

\begin{tabular}{lccccccccccccc}
\hline \multirow{2}{*}{$N$} & \multicolumn{4}{c}{$V_{n}\left(10^{3} \mathrm{kN}\right)$} & \multicolumn{3}{c}{$M_{n,}\left(10^{3} \mathrm{kN} \cdot \mathrm{m}\right)$} & \multicolumn{3}{c}{$P_{n}\left(10^{3} \mathrm{kN}\right)$} & \multicolumn{3}{c}{$k\left(10^{3} \mathrm{kN} / \mathrm{m}\right)$} \\
& & FEM & Analytical & Diff (\%) & FEM & Analytical & Diff (\%) & FEM & Analytical & Diff $(\%)$ & FEM & Analytical & Diff $(\%)$ \\
\hline 8 & $0.5 b_{w}$ & 6.28 & 6.15 & +2.1 & 105 & 106 & -1.0 & 169 & 144 & +14.9 & 3,563 & 3,767 & -5.7 \\
8 & $0.45 b_{w}$ & 6.84 & 6.74 & +1.5 & 114 & 108 & +5.6 & 176 & 149 & +15.3 & 3,808 & 3,754 & +1.4 \\
10 & $0.45 b_{w}$ & 6.56 & 6.15 & +6.3 & 114 & 106 & +7.4 & 169 & 143 & +15.4 & 3,656 & 4,081 & -11.6 \\
12 & $0.33 b_{w}$ & 7.59 & 7.29 & +4.0 & 126 & 109 & +13.3 & 182 & 153 & +15.6 & 3,833 & 4,057 & -5.8 \\
16 & $0.26 b_{w}$ & 7.12 & 6.41 & +10 & 115 & 110 & +4.2 & 186 & 156 & +16.3 & 4,104 & 4,143 & -1.0 \\
2 & $0.21 b_{w}$ & 9.57 & 9.33 & +2.5 & 138 & 114 & +16.5 & 200 & 170 & +15.1 & 4,545 & 4,551 & -0.1 \\
\hline
\end{tabular}

the one-layer embedment profile investigation. Results from the FEM and analytical model are summarized in Table 8 with configurations ranked by strength obtained from the FEM, where a positive sign in the difference represents a conservative estimation of strength by the analytical model. While sometimes overestimating the lateral stiffness, the analytical model generally yields a conservative estimate of the strength capacities. This can be attributed to the wall geometries in the FEM being fully fixed at the bottom of the footings, resulting in a not fully constrained boundary condition at the bottom of the wall sections. Furthermore, the model assumes that concrete does not have tensile resistance and that concrete crushes at $\varepsilon_{c}=0.003$, along with the approximation of parabolic stress distribution. An approximately constant 15\% difference in axial capacity can be observed, attributable to the maximum concrete stress taken as $0.85 f_{c}^{\prime}$. Nevertheless, the analytical model generally maintains a similar rank in strength, which can be useful in the design stage. Overall, accuracy of the strength model is deemed acceptable to perform further investigations that would be time-consuming using FEM, in particular for the MDOF application presented in Section 4.2.

4.2. Motion-Strength Trade-offs. The performance of the TLWD versus both motion and strength is investigated under a wide range of configurations using the validated analytical strength model. Under each configuration, the embedded TLMCD is tuned to minimize the maximum transfer function (i.e., keeping both $\left.H_{1}\right|_{\max }$ and $\left.H_{2}\right|_{\max }$ approximately equal), here assembled under the performance metric $\left.H_{1,2}\right|_{\text {max }}$. From the findings in Section 4.1, the study is limited to single-layer and single-TLMCD arrangements. The axial-bending strength $M_{n}$ is obtained at the balanced axial-bending interaction $\rho_{\text {axial }}=0.36$. Figure 11 plots the results of the multiconfiguration investigation, where a reduction in $\left.H_{1,2}\right|_{\max }$ in Figure 11 (a) is a desired feature, while that of the strength in Figures 11(b)$11(\mathrm{e})$ is not. One can observe an obvious trade-off between motion and strength performance. However, the gain in motion reduction is marginally decreasing while the loss in strength is marginally increasing with $N$ and $D b_{w}$ increasing, showing that motion performance can be substantially increased through design of the TLWD system while minimizing the adverse effects on strength. Shear strength $V_{n}$ is the most sensitive to variations in configurations, with a total variation of $47 \%$ in strength amongst all configurations, while other strength components $M_{n}, P_{n}$, and $k$ only vary maximally by $12 \%, 24 \%$, and $26 \%$ respectively.

4.3. Design Considerations. Results from the parametric investigation presented in Section 4.2, along with the extended investigation conducted using the validated strength model, point to the following considerations in designing a TLWD system. First, mitigation performance is generally achieved by adding liquid mass in the TLWD and implementing TLMCD designs with longer horizontal distances between opposite columns yields better performance. Second, in order to satisfy strength requirements, the TLMCD system should be arranged in a single layer within the wall if possible because distributing liquid mass along the width of the wall has a significant adverse effect on shear and axialbending strength. Third, the use of many smaller columns is advantageous over fewer, larger columns in the constrained TLWD environment with respect to strength, except for axial strength due to the provoked decrease in the concrete cross-section area over similar mitigation performance. Fourth, the proposed analytical strength model could be used to quickly investigate various design configurations, whereas the model will generally yield conservative estimates with respect to strength. Fifth, there is a trivial trade-off between motion and strength performance in designing a TLWD, but altering the geometry of the TLMCD does not affect both motion and strength performance proportionally, therefore pointing towards an equilibrium point depending on the design requirements. Sixth, when tuning the geometry of the TLMCD system, one must pay particular attention to the shear strength, as it is highly sensitive to the 


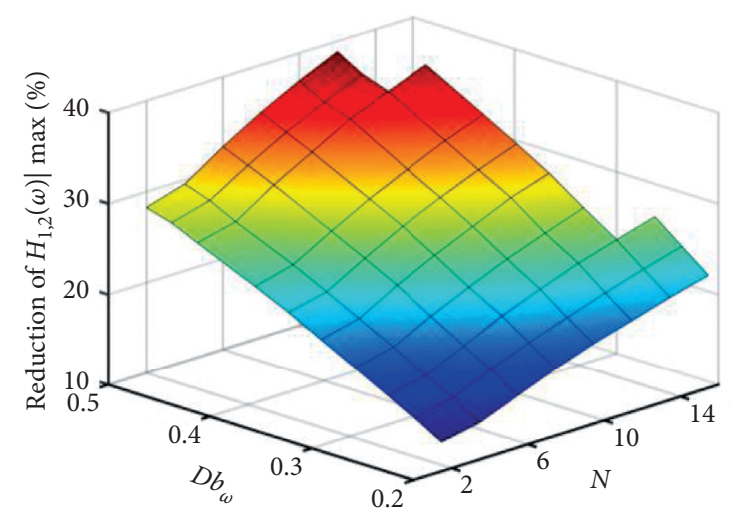

(a)

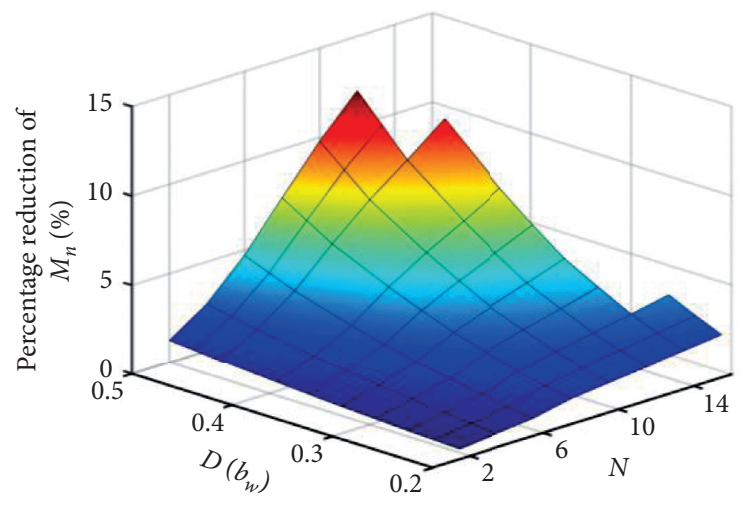

(c)

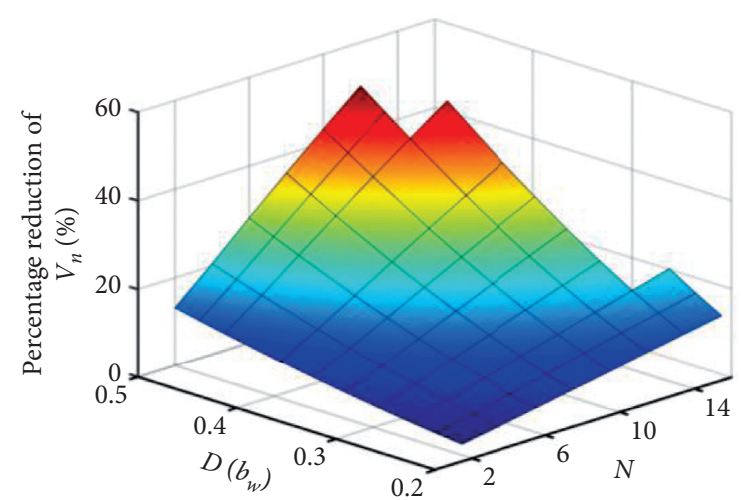

(b)

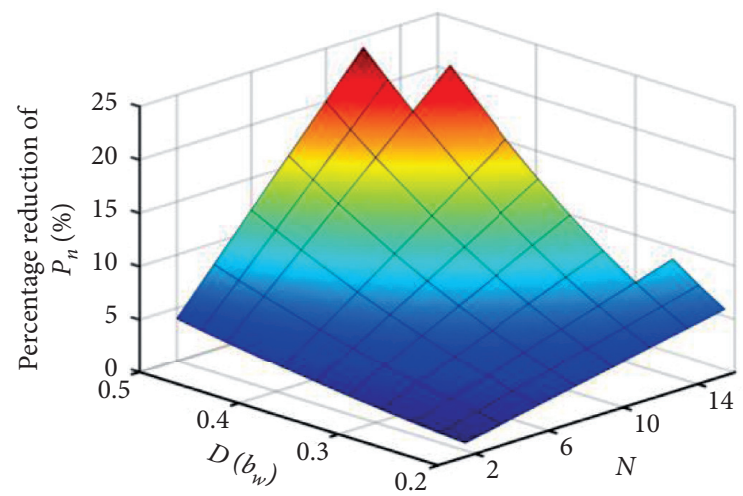

(d)

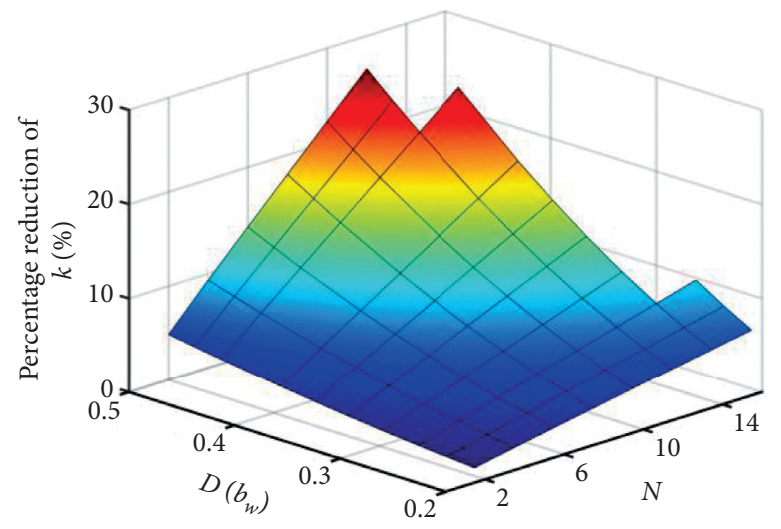

(e)

FIGURE 11: Surface plot of percentage reduction in performance objectives: (a) maximum of displacement/acceleration transfer functions, (b) nominal shear strength, (c) nominal flexural strength under balanced axial interaction, (d) nominal axial strength, and (e) lateral stiffness.

selected configuration and constitute a key design property in the design of a shear wall.

\section{High-Rise Building Example}

In this section, the performance of the TLWD is numerically validated on a realistic high-rise building subject to stochastic wind excitations. Motion and strength performance is assessed, with strength analyzed using the proposed analytical model.
5.1. Numerical Model. A 42-story RC residential building (Building 1A) [29] is used for the simulations. It consists of six centrally located core walls along east-west direction surrounded on the perimeter by concrete columns. The walls have constant lengths, but their width varies along the height of the structure. Geometries and material properties and reinforcement details can be found in literature [29]. Here, the east-west direction of the building is simulated as a lumped-mass shear system. The fundamental damping ratio of the building is taken as $2 \%$ with stiffness proportional 
TABLE 9: Dynamic properties of 42-story building.

\begin{tabular}{|c|c|c|c|c|c|c|c|}
\hline Floor & Height $(\mathrm{m})$ & Mass $\left(10^{3} \mathrm{~kg}\right)$ & Stiffness $\left(10^{3} \mathrm{kN} / \mathrm{m}\right)$ & Floor & Height $(\mathrm{m})$ & Mass $\left(10^{3} \mathrm{~kg}\right)$ & Stiffness $\left(10^{3} \mathrm{kN} / \mathrm{m}\right)$ \\
\hline 1 & 4.16 & 1189 & 11,492 & 22 & 3.25 & 893 & 946 \\
\hline 2 & 3.25 & 1099 & 7,861 & 23 & 3.25 & 893 & 883 \\
\hline 3 & 3.25 & 1099 & 6,225 & 24 & 3.25 & 893 & 821 \\
\hline 4 & 3.25 & 1099 & 4,532 & 25 & 3.25 & 843 & 762 \\
\hline 5 & 3.25 & 1099 & 3,677 & 26 & 3.25 & 843 & 741 \\
\hline 6 & 3.25 & 1041 & 3,155 & 27 & 3.25 & 843 & 721 \\
\hline 7 & 3.25 & 1041 & 2,797 & 28 & 3.25 & 843 & 702 \\
\hline 8 & 3.25 & 1041 & 2,536 & 29 & 3.25 & 810 & 685 \\
\hline 9 & 3.25 & 1041 & 2,336 & 30 & 3.25 & 810 & 669 \\
\hline 10 & 3.25 & 1041 & 2,177 & 31 & 3.25 & 810 & 654 \\
\hline 11 & 3.25 & 1000 & 1,970 & 32 & 3.25 & 810 & 640 \\
\hline 12 & 3.25 & 1000 & 1,784 & 33 & 3.25 & 810 & 624 \\
\hline 13 & 3.25 & 1000 & 1,615 & 34 & 3.25 & 777 & 609 \\
\hline 14 & 3.25 & 1000 & 1,460 & 35 & 3.25 & 777 & 594 \\
\hline 15 & 3.25 & 1000 & 1,315 & 36 & 3.25 & 777 & 580 \\
\hline 16 & 3.25 & 934 & 1,256 & 37 & 3.25 & 777 & 567 \\
\hline 17 & 3.25 & 934 & 1,205 & 38 & 3.25 & 777 & 557 \\
\hline 18 & 3.25 & 934 & 1,159 & 39 & 3.25 & 777 & 547 \\
\hline 19 & 3.25 & 934 & 1,118 & 40 & 3.25 & 777 & 538 \\
\hline 20 & 3.25 & 934 & 1,081 & 41 & 3.25 & 777 & 530 \\
\hline 21 & 3.25 & 893 & 1,012 & 42 & 3.25 & 872 & 521 \\
\hline
\end{tabular}

damping. The dynamic properties of the building used in the simulation are tabulated in Table 9.

The simulated wind speed consists of two components, the steady mean speed $V_{m}$ and the fluctuating speed $V_{f}$, due to aerodynamic turbulence:

$$
V(z, t)=V_{m}(z)+V_{f}(z, t) .
$$

The steady mean speed is determined based on the wind profile power law [30] as follows:

$$
\begin{aligned}
& V_{\text {terrain }}=V_{0} \frac{v_{*}}{v_{0}} \frac{\ln \left(10 / z_{*}\right)}{\ln \left(10 / z_{0}\right)}, \\
& V_{m}(z)=V_{\text {terrain }} \frac{\ln \left(z / z_{*}\right)}{\ln \left(10 / z_{*}\right)},
\end{aligned}
$$

where $V_{0}$ is the 3-second gust speed obtained from hazard maps in ASCE 7-16 [31], $V_{\text {terrain }}$ is the mean velocity for a given terrain, $v_{*}$ and $v_{0}$ are the shear velocities of the building site and open terrain, and $z_{*}$ and $z_{0}$ are the surface roughness for the building site and open terrain, respectively. A multivariate stochastic Gaussian process with crossspectral density function is used for modeling turbulence [32] as follows:

$$
\begin{gathered}
S_{i j}(\Omega)= \begin{cases}S_{k}\left(z_{i}, \Omega\right), & i=j, \\
\sqrt{S_{k}\left(z_{i}, \Omega\right) S_{k}\left(z_{j}, \Omega\right)} C_{i j}\left(z_{i}, z_{j}, \Omega\right), & i \neq j\end{cases} \\
S_{k}(z, \Omega)=\frac{50}{\pi} v_{*}^{2} \frac{z}{V_{m}(z)} \frac{1}{\left(1+50(\Omega z / 2 \pi V(z))^{(5 / 3)}\right.},
\end{gathered}
$$

where $S_{k}$ is the Kaimal power spectral density function in along-wind direction, $O$ is the excitation frequency, and $z$ is the height of floors. The coherence function between $i$ th and $j$ th locations in equation (32) is defined as

$$
C_{i j}(\Omega)=\exp \left(-\frac{10 \Omega \Delta z}{\pi\left(V_{m}\left(z_{i}\right)+V\left(z_{j}\right)\right)}\right) .
$$

The cross-spectral density matrix, $\mathbf{S}(\Omega)$, containing cross-spectral density functions at different heights, is decomposed using Cholesky's decomposition:

$$
\mathbf{S}(\Omega)=\mathbf{H}(\Omega) \mathbf{H}^{* T}(\Omega),
$$

where $\mathbf{H}(\Omega)$ is a lower triangular matrix with generally complex off-diagonal elements. Once the matrix is decomposed, the stochastic fluctuating wind speed $V_{f}\left(z_{i}, t\right)$ can be obtained by the following series:

$$
\begin{aligned}
V_{f}\left(z_{i}, t\right)= & 2 \sum_{k=1}^{n} \sum_{l=1}^{N_{w}}\left|H_{i k}\left(\Omega_{k l}\right)\right| \\
& \cdot \sqrt{\Delta \Omega} \cos \left(\Omega_{l k} t-\theta_{i k}\left(\Omega_{k l}\right)+\Phi_{k l}\right),
\end{aligned}
$$

where $\Phi_{k l}$ is the random phase ranged from 0 to $2 \pi$; the phase $\theta_{i k}\left(\Omega_{k l}\right)$ and the double-indexing frequency $\Omega_{k l}$ is given by

$$
\begin{aligned}
\theta_{i k}\left(\Omega_{k l}\right) & =\tan ^{-1}\left(\frac{\operatorname{Im}\left(H_{i k}\left(\Omega_{k l}\right)\right)}{\operatorname{Re}\left(H_{i k}\left(\Omega_{k l}\right)\right)}\right), \\
\Omega_{k l} & =\left(l-\frac{N_{\Omega}-l}{N_{\Omega}}\right) \Delta \Omega, \\
\Delta \Omega & =\frac{\Omega_{c}}{N_{\Omega}}
\end{aligned}
$$

where $\Omega_{c}$ is an upper cutoff frequency and $N_{\Omega}$ is the total number of random frequency points. 


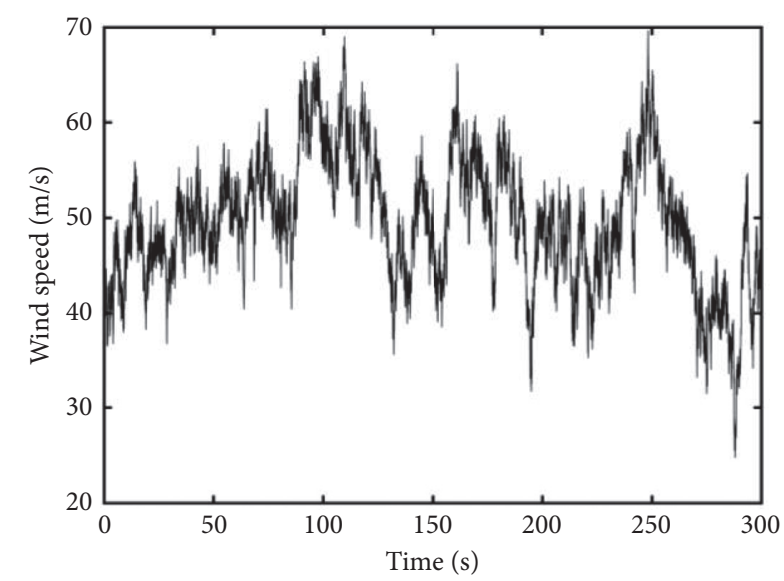

(a)

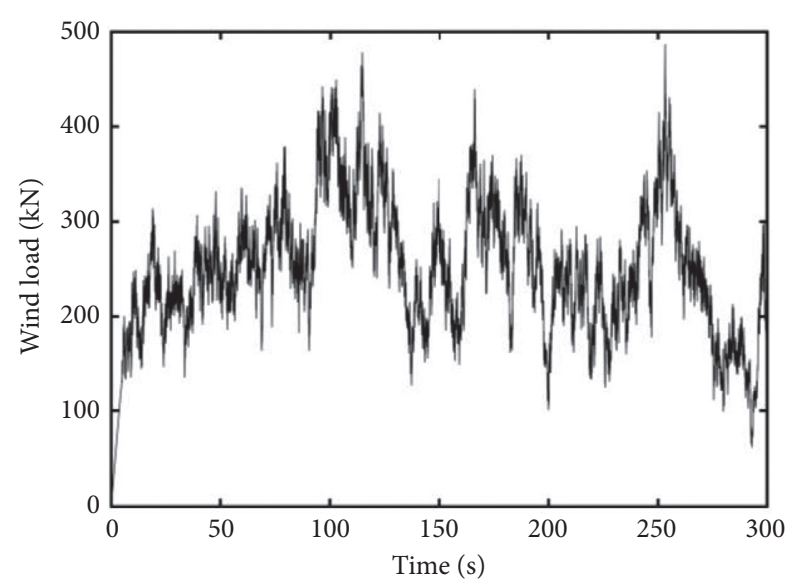

(b)

Figure 12: Typical wind hazard realization at the top floor of the building: (a) wind speed and (b) wind load time series.

The wind load is converted from wind speed to concentrated forces applied on the building floors [30] using

$$
F_{w}(z, t)=\frac{1}{2} \rho V^{2}(z, t) A_{p} C_{D}
$$

where $\rho$ is the air density, $A_{p}$ is the projected area exposed to the wind pressure, and $C_{D}$ is the drag coefficient of the structure. Across-wind forces and vortex shedding are neglected for simplicity, and it is assumed that the alongwind response of the structure dominates motion. A total of 20 wind realizations are produced taking a suburban region based on occupancy category II (residential building) [31] with $7 \%$ probability of exceedance in 50 years. Figure 12 plots a typical wind realization speed $V$ (Figure 12(a)) and associated load $F_{w}$ (Figure 12(b)) time histories occurring at the top floor of the building using the input parameters listed in Table 10.

5.2. TLWD Design. In the following numerical investigations, three optimally tuned TLWD configurations are considered: TLWD1 has a 2-column TLMCD of column cross-section diameter $D=0.5 b_{w}$, TLWD2 has a 6 -column TLMCD of column cross-section diameter $D=0.5 b_{w}$, and TLWD3 has a 12-column TLMCD of column cross-section diameter $D=0.37 b_{w}$. TLWD1 is selected to mimic a distributed TLCDs system, TLWD2 is selected to improve on TLWD1 through added inertia, and TLWD3 is selected from Table 5 and to perform similar to TLWD2 for motion mitigation, yielding minimal strength reduction. The simplified strength model is used to calculate the strength reduction in parameters $V_{n}, M_{n}, P_{n}$, and $k$ caused by the integration of the TLMCDs. In this numerical example, TLWDs are vertically distributed within the top 20 floors (from the 23rd to the 42nd floor inclusively). The shear wall parameters including the wall width $b_{w}$, longitudinal reinforcing ratio $\rho_{w}$, and transverse reinforcing ratio $\rho_{t}$ are listed in Table 11 along with the resulting reductions in strength.

A comparison of strength reductions shows that TLWD1 yields a significantly stronger shear wall, except for the axial-
TABle 10: Parameters for the stochastic wind model.

\begin{tabular}{lcc}
\hline Parameters & Value & Unit \\
\hline$V_{0}$ & 49 & $\mathrm{~m} / \mathrm{s}$ \\
$z_{0}$ & 0.07 & - \\
$z^{*}$ & 0.3 & - \\
$\Omega_{c}$ & $20 \pi$ & $\mathrm{rad} / \mathrm{s}$ \\
$N_{\Omega}$ & $2^{13}$ & - \\
$\rho_{\text {air }}$ & 1.225 & $\mathrm{~kg} / \mathrm{m}^{3}$ \\
$C_{D}$ & 1.4 & - \\
\hline
\end{tabular}

bending strength compared with TLWD3. However, the effect of embedding a TLMCD on the axial-bending strength is not substantial, with a maximum reported reduction of $5.1 \%$. Configuration TLWD3 does yield an improvement in strength relative to TLWD2. Nevertheless, the differences in strength reductions between both configurations are all within 3.6\%. Overall, adding more column cross-section areas within the shear wall (TLWD1 versus TLWD2) does have an adverse effect on strength, while distributing the cross-section areas with the wall (TLWD2 versus TLWD3) improves on strength, consistent with findings in Section 5.1.

5.3. Motion Performance. For the numerical investigation on motion performance, it is assumed that all TLWDs have identical geometries and dynamic properties, and they are modeled as a single equivalent TLWD at each floor, with a maximum of three TLMCDs per interstory wall. The placement of TLWD is first investigated using TLWD2 configuration and selected because it adopts the geometry of a TLCD (TLWD1) but with added inertia. Four vertical distributions are considered, with the TLWDs installed over the top 5, top 10, top 15, and top 20 floors. Figure 13 plots the fitted normal distributions of the maximum structural responses under the 20 wind realizations. Table 12 lists the average drift $\mu_{d}$ and acceleration responses $\mu_{a}$, the standard deviation of the drift $\sigma_{d}$ and acceleration responses $\sigma_{a}$, and the probability of exceeding the acceleration threshold $P_{a}$. Results show that, for this particular building, increasing the 
TABLE 11: Strength reduction (\%) of the TLWDs compared to the original wall design.

\begin{tabular}{|c|c|c|c|c|c|c|c|c|c|c|c|c|c|c|c|}
\hline \multirow{2}{*}{ Floor } & \multirow{2}{*}{$b_{w}(\mathrm{~mm})$} & \multirow{2}{*}{$\rho_{w}(\%)$} & \multirow{2}{*}{$\rho_{t}(\%)$} & \multicolumn{4}{|c|}{ TLWD1 } & \multicolumn{4}{|c|}{ TLWD2 } & \multicolumn{4}{|c|}{ TLWD3 } \\
\hline & & & & $V_{n}$ & $M_{n}$ & $P_{n}$ & $k$ & $V_{n}$ & $M_{n}$ & $P_{n}$ & $k$ & $V_{n}$ & $M_{n}$ & $P_{n}$ & $k$ \\
\hline $23-25$ & 610 & 2.4 & 0.9 & 23.9 & 4.9 & 6.1 & 15.1 & 38.3 & 4.9 & 18.7 & 24.4 & 36.2 & 3.5 & 20.5 & 23.9 \\
\hline $26-30$ & 533 & 2.7 & 0.6 & 26.1 & 5.1 & 7.2 & 17.3 & 45.0 & 5.1 & 21.5 & 27.9 & 41.4 & 4.2 & 23.2 & 27.0 \\
\hline $31-36$ & 533 & 1.8 & 0.6 & 26.1 & 4.2 & 7.4 & 17.3 & 45.0 & 4.2 & 22.1 & 27.9 & 41.4 & 2.7 & 23.9 & 27.0 \\
\hline $37-42$ & 533 & 1.4 & 0.6 & 26.1 & 3.4 & 7.4 & 17.3 & 45.0 & 3.4 & 22.2 & 27.9 & 41.4 & 2.1 & 24.1 & 27.0 \\
\hline
\end{tabular}

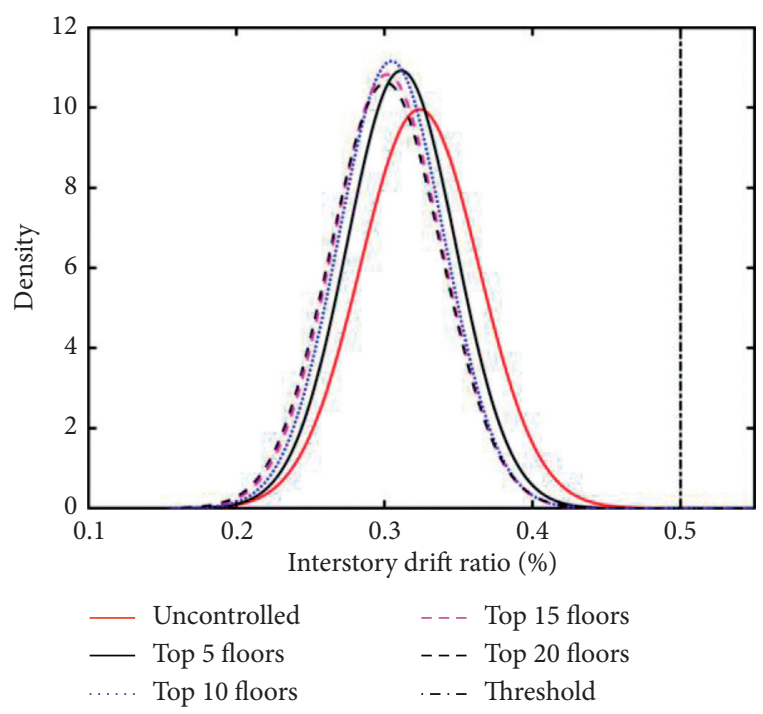

(a)

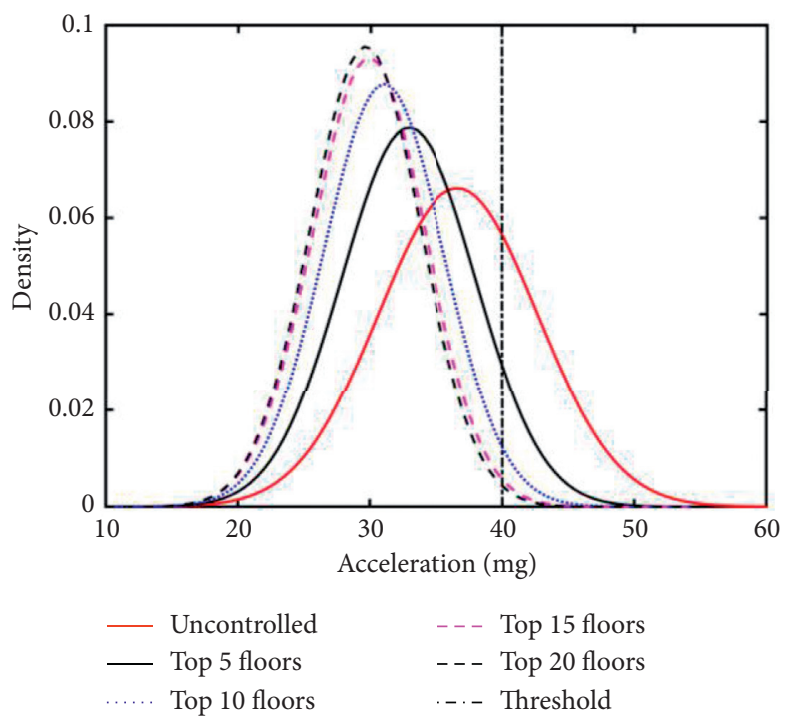

(b)

FiguRE 13: Distribution of maximum absolute responses under various vertical TLWD arrangements: (a) interstory drift ratio and (b) acceleration, with the dashed vertical line showing the performance threshold.

TABle 12: Performance of TLWD2 under various vertical arrangements.

\begin{tabular}{lccccc}
\hline & $\mu_{d}(\mathrm{~mm})$ & $\sigma_{d}(\mathrm{~mm})$ & $\mu_{a}(\mathrm{mg})$ & $\sigma_{a}(\mathrm{mg})$ & $P_{a}(\%)$ \\
\hline Uncontrolled & 10.7 & 1.3 & 36.6 & 6.02 & 28.6 \\
Top 5-floor & 10.1 & 1.2 & 32.9 & 5.06 & 8.03 \\
Top 10-floor & 10.1 & 1.2 & 31 & 4.55 & 2.4 \\
Top 15-floor & 9.8 & 1.2 & 29.9 & 4.28 & 0.9 \\
Top 20-floor & 9.8 & 1.2 & 29.6 & 4.18 & 0.6 \\
\hline
\end{tabular}

number of TLWDs over more floors yields marginally decreasing gains in acceleration mitigation and the utilization of TLWDs does not significantly affect interstory drift. In the case of acceleration control, the use of TLWDs over at least the last ten floors is necessary in ensuring that the structure does not significantly exceed the acceleration threshold of $40 \mathrm{mg}$, where $40 \mathrm{mg}$ corresponds to an acceptable motion performance for essential structures under rare winds [33]. It can also be noted that performance under drift is always satisfied, even in the uncontrolled case. From these results, arrangement of TLWDs over the top 15 floors is selected to continue the numerical investigation.

Mitigation performance for TLWDs1-3 is now assessed against that of a conventional TLCD installed at the top of the building. To enable fair comparison, the TLCD's capacity is equal to that of TLWD1 by constraining its geometry, where its horizontal length is equals to that of one embedded TLMCD, and the area of its vertical columns is equal to the sum of all of the single column areas of each TLMCD. Note that such TLCD geometry may not be practical and is simply used to benchmark performance. Figure 14 plots the fitted normal distributions of the maximum structural responses under the 20 wind realizations, and Figure 15 plots the response profiles using the averaged maximum structural responses. Table 13 lists the average drift $\mu_{d}$ and acceleration responses $\mu_{a}$, the standard deviation of the drift $\sigma_{d}$ and acceleration responses $\sigma_{a}$, and the probability of exceeding the acceleration threshold $P_{a}$.

Results show that configurations TLWD2 and TLWD3 successfully reduce the mean maximum acceleration of the uncontrolled case by $18 \%$ and slightly outperform the TLCD case by approximate $3 \%$, yet not necessitating large space at top of the building. A cross-comparison amongst TLWDs reveals that TLWD2 and TLWD3 slightly outperform TLWD1 by approximate $4.2 \%$ in terms of acceleration mitigation. However, this improvement in mitigation keeps the structure's maximum acceleration motion under the performance threshold at least $99 \%$ of the time. An 


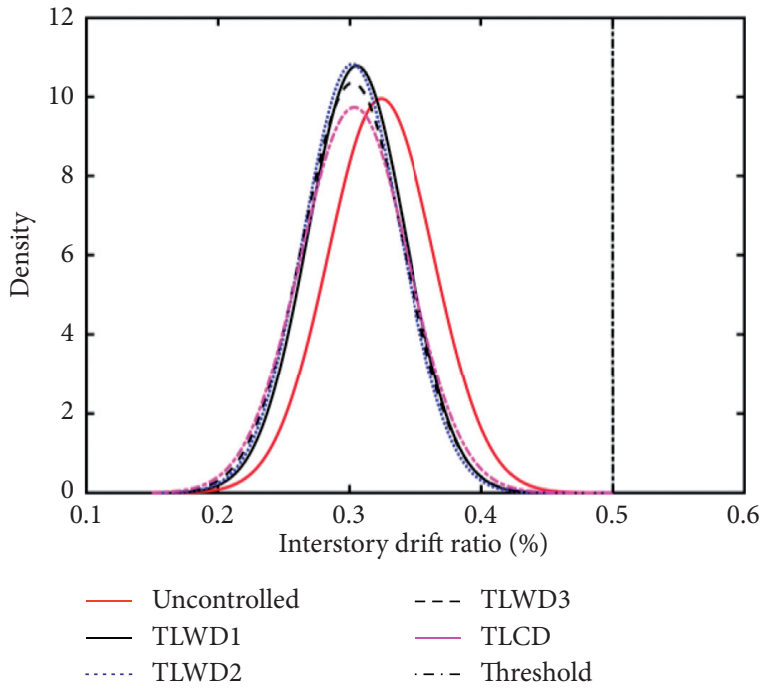

(a)

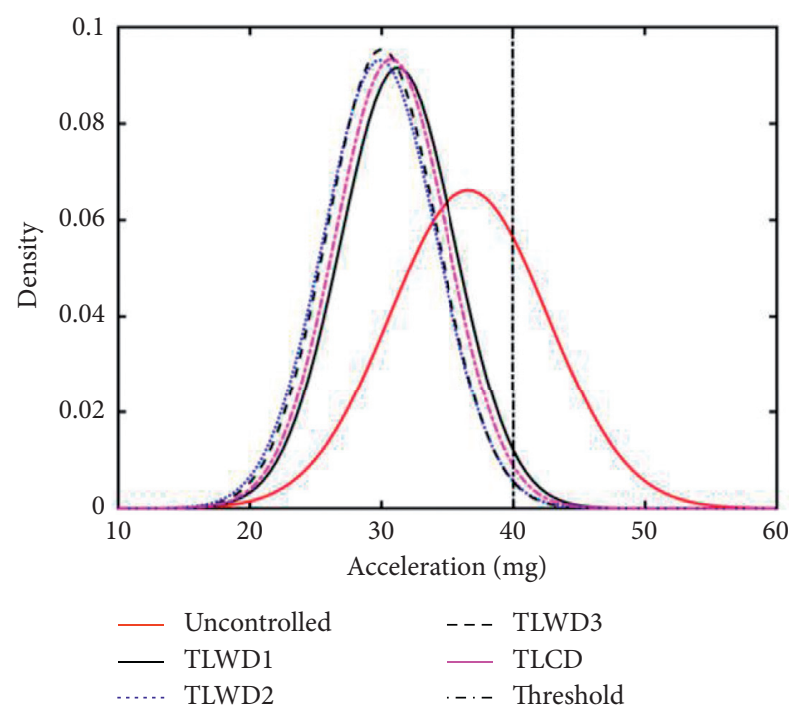

(b)

FigURE 14: Distribution of maximum absolute responses using TLWDs arranged over the last 15 floors: (a) interstory drift ratio and (b) acceleration.

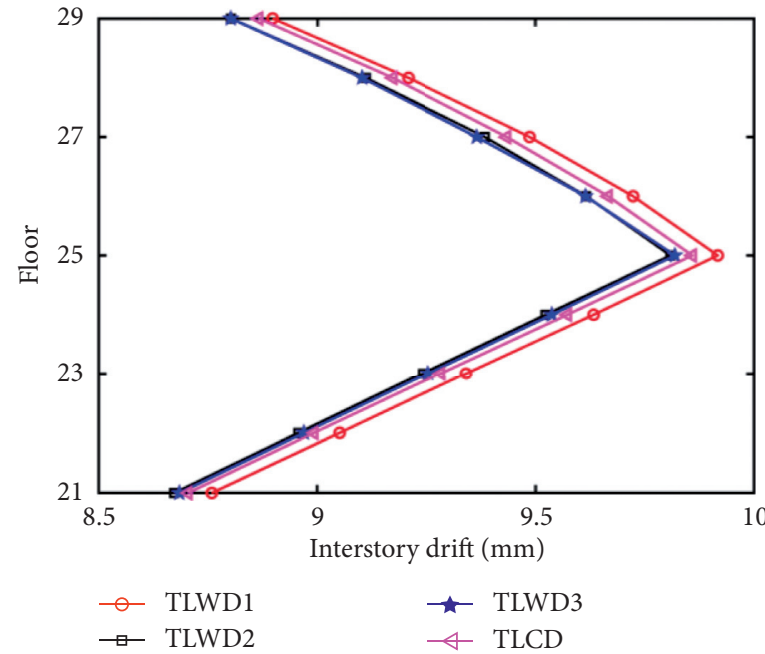

(a)

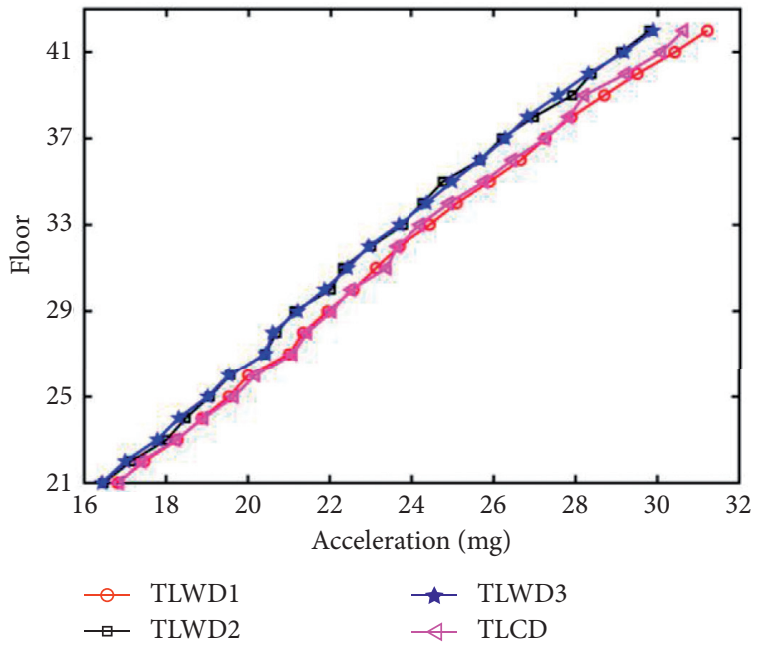

(b)

Figure 15: Mean maximum floor response profiles for TLWDs installed over the last 15 floors: (a) zoom on maximum interstory drift and (b) maximum acceleration over top 20 floors.

TABLE 13: Motion performance for TLWDs installed over the last 15 floors.

\begin{tabular}{lccccc}
\hline & $\mu_{d}(\mathrm{~mm})$ & $\sigma_{d}(\mathrm{~mm})$ & $\mu_{a}(\mathrm{mg})$ & $\sigma_{a}(\mathrm{mg})$ & $P_{a}(\%)$ \\
\hline Uncontrolled & 10.7 & 1.3 & 36.6 & 31.2 & 4.35 \\
TLWD1 & 10.1 & 1.2 & 29.9 & 4.28 & 2.2 \\
TLWD2 & 9.8 & 1.2 & 30 & 4.18 & 0.9 \\
TLWD3 & 9.8 & 1.2 & 30.8 & 4.27 \\
TLCD & 10.1 & 1.2 & 0.8 \\
\hline
\end{tabular}


inspection of the floor drift and acceleration profiles also shows that TLWD2 and TLWD3 outperform TLWD1 and the TLCD. This enhancement in performance, in particular, with respect to TLWD1, is consistent with the findings in Section 5.2.

\section{Conclusion}

In this study, a multifunctional shear wall, termed tuned liquid wall damper (TLWD), capable of vibration mitigation was investigated. The TLWD consists of a tuned liquid multiple column damper (TLMCD) system integrated within a reinforced concrete shear wall component. The objective of the paper was to evaluate trade-offs between motion and strength performance in designing a TLWD. To empower fast evaluation of strength properties on multidegrees-of-freedom representations, a simplified analytical strength model was proposed based on ACI 318-14 and validated against finite element model simulations conducted on the simplified representation of a 42-story building. The finite element analysis combined with numerical simulations of the simplified representation was used to establish design considerations. In particular, it was found that distributing liquid mass among a higher number of single columns over a single-layer configuration was preferable in minimizing the reduction in strength while providing enhanced mitigation.

After that, numerical simulations were conducted on a more realistic representation of the 42-story building subjected to 20 stochastic wind loads. First, TLWDs were sequentially added starting from the top floor of the structure to address the mitigation performance on the vertical distribution of TLWDs. The results showed a decreasing marginal gain in mitigation performance. Further simulations were conducted comparing three different TLMCD configurations installed on the last 15 floors. Results showed that adding liquid inertia through additional columns (TLWD1 versus TLWD2) decreased strength while increasing mitigation capabilities and distributing the liquid inertia through additional, smaller columns (TLWD2 versus TLWD3) increased strength while also increasing mitigation capabilities, thus confirming design considerations established through the simplified representation. The use of TLWDs showed important gain in mitigation relative to an uncontrolled case, in particular, for acceleration mitigation. TLWDs also outperformed, yet not significantly, a geometrically constrained TLCD installed at the top of the structure occupying a notably larger space. The use of a distributed liquid inertia (TLWD3) exhibited the best mitigation performance.

Overall, this study demonstrated the potential of the TLWD system as a passive energy mitigation system. While a distributed TLWD system utilizes a higher total liquid mass compared to a conventional TLCD installed at the top floor, its advantage resides in constraining the energy dissipation system with an existing structural component, therefore eliminating the need to utilize a dedicated area in the build as long as structural strength can be ensured. The proposed TLWD is practically feasible due to the low maintenance requirements, cost-effectiveness, and high mechanical robustness characteristics of TLMCDs. The performance of the TLWD system could be potentially improved by optimizing designs at each floor or by integrating semiactive capabilities by actively controlling the orifice block ratios. These investigations are left to future work.

\section{Data Availability}

The data that support the findings of this study are available from the corresponding author upon request.

\section{Disclosure}

Any opinions, findings, and conclusions or recommendations expressed in this study are those of the authors and do not necessarily reflect the views of the National Science Foundation.

\section{Conflicts of Interest}

The authors declare no conflicts of interest.

\section{Acknowledgments}

This work was supported in part by National Science Foundation under Grant no. CMMI-1562992.

\section{References}

[1] J. Connor and S. Laflamme, Structural Motion Engineering, Springer, Berlin, Germany, 2014.

[2] T. E. Saaed, G. Nikolakopoulos, J.-E. Jonasson, and H. Hedlund, "A state-of-the-art review of structural control systems," Journal of Vibration and Control, vol. 21, no. 5, pp. 919-937, 2013.

[3] F. Ubertini, "Prevention of suspension bridge flutter using multiple tuned mass dampers," Wind and Structures An International Journal, vol. 13, no. 3, pp. 235-256, 2010.

[4] Y. Saoka, F. Sakai, S. Takaeda, and T. Tamaki, "On the suppression of vibrations by tuned liquid column dampers," in Proceedings of the Annual Meeting of JSCE, Tokyo, Japan, September 1988.

[5] F. Sakai, S. Takaeda, and T. Tamaki, "Tuned liquid column damper-new type device for suppression of building vibration," in Proceedings of the International Conference on HighRise Building, pp. 926-931, Nanjing, China, August 1989.

[6] N. Cavalagli, C. Biscarini, A. L. Facci, F. Ubertini, and S. Ubertini, "Experimental and numerical analysis of energy dissipation in a sloshing absorber," Journal of Fluids and Structures, vol. 68, pp. 466-481, 2017.

[7] Y. L. Xu, B. Samali, and K. C. S. Kwok, "Control of along-wind response of structures by mass and liquid dampers," Journal of Engineering Mechanics, vol. 118, no. 1, pp. 20-39, 1992.

[8] B. F. Spencer and S. Nagarajaiah, "State of the art of structural control," Journal of Structural Engineering, vol. 129, no. 7, pp. 845-856, 2003.

[9] H. Gao, K. C. S. Kwok, and B. Samali, "Optimization of tuned liquid column dampers," Engineering Structures, vol. 19, no. 6, pp. 476-486, 1997.

[10] C. C. Chang and W. L. Qu, "Unified dynamic absorber design formulas for wind-induced vibration control of tall 
buildings," The Structural Design of Tall Buildings, vol. 7, no. 2, pp. 147-166, 1998.

[11] A. Y. J. Won, J. A. Pires, and M. A. Haroun, "Stochastic seismic performance evaluation of tuned liquid column dampers," Earthquake Engineering \& Structural Dynamics, vol. 25, no. 11, pp. 1259-1274, 1996.

[12] S. K. Yalla and A. Kareem, "Optimum absorber parameters for tuned liquid column dampers," Journal of Structural Engineering, vol. 126, no. 8, pp. 906-915, 2000.

[13] L. Cao, Y. Gong, F. Ubertini, H. Wu, A. Chen, and S. Laflamme, "Development and validation of a nonlinear dynamic model for tuned liquid multiple columns dampers," Journal of Sound and Vibration, vol. 487, Article ID 115624, 2020.

[14] L. Ye, X. Lu, Z. Qu, and J. Hou, "Distributed TLDs in RC floors and their vibration reduction efficiency," Earthquake Engineering and Engineering Vibration, vol. 7, no. 1, pp. 107-112, 2008.

[15] Y. Matia and A. D. Gat, "Dynamics of elastic beams with embedded fluid-filled parallel-channel networks," Soft Robotics, vol. 2, no. 1, pp. 42-47, 2015.

[16] H. Guan, C. Cooper, and D.-J. Lee, "Ultimate strength analysis of normal and high strength concrete wall panels with varying opening configurations," Engineering Structures, vol. 32, no. 5, pp. 1341-1355, 2010.

[17] H. Wu, A. Chen, and S. Laflamme, "Seismic behavior of glass fiber-reinforced polymer wall panels," Composite Structures, vol. 203, pp. 300-309, 2018.

[18] L. M. Massone, G. Muñoz, and F. Rojas, "Experimental and numerical cyclic response of RC walls with openings," Engineering Structures, vol. 178, pp. 318-330, 2019.

[19] I. E. Idelchik, Handbook of Hydraulic Resistance, Hemisphere Publishing Corp., Washington, DC, USA, 1986.

[20] ACI 318-14, Building Code Requirements for Structural Concrete: Commentary on Building Code Requirements for Structural Concrete (ACI 318R-14), American Concrete Institute, Farmington Hills, MI, USA, 2014.

[21] S. M. Manohar, Seismic Design of RC Buildings: Theory and Practice, Springer, Berlin, Germany, 2016.

[22] J. Lubliner, J. Oliver, S. Oller, and E. Oñate, "A plastic-damage model for concrete," International Journal of Solids and Structures, vol. 25, no. 3, pp. 299-326, 1989.

[23] J. Lee and G. L. Fenves, "Plastic-damage model for cyclic loading of concrete structures," Journal of Engineering Mechanics, vol. 124, no. 8, pp. 892-900, 1998.

[24] Y. Sümer and M. Aktaş, "Defining parameters for concrete damage plasticity model," Challenge Journal of Structural Mechanics, vol. 32, 2015.

[25] Dassault Systemes Simulia Corp, Abaqus/CAE User's Manual, Dassault Systemes Simulia Corp, Providence; RI: USA, 2014.

[26] L. S. Hsu and C.-T. T. Hsu, "Complete stress - strain behaviour of high-strength concrete under compression," Magazine of Concrete Research, vol. 46, no. 169, pp. 301-312, 1994.

[27] R. Nayal and H. A. Rasheed, "Tension stiffening model for concrete beams reinforced with steel and FRP bars," Journal of Materials in Civil Engineering, vol. 18, no. 6, pp. 831-841, 2006.

[28] B. L. Wahalathantri, T. H. T. Chan, and A. Fawzia, "A material model for flexural crack simulation in reinforced concrete elements using abaqus," in Proceedings of the First International Conference on Engineering, Designing and Developing the Built Environment for Sustainable Wellbeing, pp. 260-264, Rome, Italy, August 2011.
[29] J. Moehle, Y. Bozorgnia, N. Jayaram et al., Case Studies of the Seismic Performance of Tall Buildings Designed by Alternative Means, Pacific Earthquake Engineering Research Center, Richmond, CL, USA, 2011.

[30] E. Simiu and R. H. Scanlan, Wind Effects on Structures: Fundamentals and Applications to Design, Dover Publications, Mineola, NY, USA, 2008.

[31] American Society of Civil Engineers, Minimum Design Loads and Associated Criteria for Buildings and Other Structures, American Society of Civil Engineers, Reston, VA, USA, 2017.

[32] G. Deodatis, "Simulation of ergodic multivariate stochastic processes," Journal of Engineering Mechanics, vol. 122, no. 8, pp. 778-787, 1996.

[33] L. Micheli, A. Alipour, S. Laflamme, and P. Sarkar, "Performance-based design with life-cycle cost assessment for damping systems integrated in wind excited tall buildings," Engineering Structures, vol. 195, pp. 438-451, 2019. 